\title{
Bounds for the spectrum of compact linear operators in a preordered Banach space
}

\author{
Andrei Rontó
}




\title{
BOUNDS FOR THE SPECTRUM OF COMPACT LINEAR OPERATORS IN A PREORDERED BANACH SPACE
}

\author{
ANDREI RONTÓ
}

[Received: December 20, 2005]

\begin{abstract}
Aвstract. We establish new efficient upper bounds for the spectral radius of a completely continuous operator in a Banach space equipped by a suitable preordering. The operator considered is assumed to admit a majorant preserving the preordering and, generally speaking, may not leave the given wedge invariant.
\end{abstract}

Mathematics Subject Classification: 46B40, 34K30, 46N20

Keywords: Wedge, cone, spectral radius, eigenvalue, upper bound

\section{Contents}

1. Introduction 54

2. Wedges in Banach spaces and linear operators leaving them invariant 55

2.1. Wedges and related preorderings $\quad 55$

2.2. Measurable elements of a Banach space 56

2.3. Strict inequalities with respect to a wedge 58

2.4. The mappings $n_{K, f}: X_{K}(f) \rightarrow[0,+\infty) \quad 60$

2.5. Complexification of a wedge and the related objects 62

2.6. Linear operators vanishing on the blade of a wedge 72

3. Upper bounds for $K$-substantial eigenvalues 73

4. A theorem on the spectral radius of a compact operator 77

5. Importance of condition (4.1) 80

6. Unique solvability of linear equations $\quad 81$

7. An example of a linear integral equation 83

7.1. The general argument deviation $\quad 83$

7.2. The case of a power transformation of argument 87

References $\quad 89$

The research was supported in part by AS CR, Institutional Research Plan AV0Z10190503, and GA CR, Grant No. 201/06/0254. A draft version of this paper had been prepared in 2003 and appeared in 2005 in the form of the preprint [12]. 


\section{InTRODUCTION}

Many concrete problems of various nature, where the question of uniqueness of a solution is essential, as is well-known, lead one to the study of regular values of a certain bounded linear operator. Since it is always natural to try to get some useful information on the base of as few initial data as possible, is appears that estimates of the spectral radius of an operator which are derived from certain relations involving its value on a single element only, should be of the best imaginable efficiency.

There is a vast literature devoted to this kind of estimates of spectra of linear operators that are positive with respect to a cone in a Banach space (see, e. g., [6,7]). The main idea of such statements, dating back to O. Perron, P. Jentzsch, P. Urysohn, L. Collatz, and M. Krein, was developed by many authors (see, e. g., $[3,6,7,10,13$, 15]).

The conditions imposed on the operator and the space where it acts vary as well as the assumed properties of the chosen element do. For example, the spectral radius of $A$ admits the estimate

$$
r(A) \geq \gamma,
$$

where $\gamma$ is a given positive constant, on the assumption [6] that $A$ is a bounded linear operator leaving invariant a cone $K$ and such that the relation $A g-\gamma g \in K$ is true for a certain element $g \in(K-K) \backslash(-K)$. On the other hand, the number $r(A)$ satisfies the inequality

$$
r(A) \leq \gamma
$$

provided that $A(K) \subseteq K, K$ is a solid normal cone, and the inclusion

$$
\gamma g-A g \in K
$$

is true for some interior element $g$ of $K$ [7]. It is natural to find out that obtaining the upper bounds for the spectral radius is more difficult, that a relation of type (1.3) implies (1.2) only under additional conditions on $A$ and $K$, and that these additional conditions are stronger than those guaranteeing a similar estimate (1.1) from below.

In this paper, we establish a new theorem of the kind indicated for linear mappings majorised by linear operators preserving a preordering which may not be a partial ordering (Section 4). More precisely, we obtain an efficient upper bound for the spectral radius of a completely continuous linear mapping $A: X \rightarrow X$ representable in the form

$$
A=A_{1}-A_{2},
$$

where $X$ is a Banach space with a wedge $K$ (which, generally speaking, may not be a cone), and the operators $A_{1}$ and $A_{2}$ leave $K$ invariant. The proof of the result mentioned (namely, Theorem 4.1) uses an inequality satisfied by the so-called $K$ substantial eigenvalues of $A$ and established in Section 3. Note that, in the theorem mentioned, the property expressing a certain "strong positivity" of a test element $g$ from the corresponding relation (1.3) depends upon the structure of the image space of the operator $A$ (see Remark 4.6). 
Theorem 4.1 is estabished under the condition that operator (1.4) is completely continuous. This assumption is not unnatural because our present study of completely continuous linear operators in a preordered Banach space is motivated mainly by the related problems arising in the theory of functional differential equations, and to a boundary value problem for a linear functional differential equation, a compact linear operator is usually associated. For example, the set of absolutely continuous solutions of the homogeneous initial value problem

$$
\begin{gathered}
u^{\prime}(t)=p(t) u(\omega(t)), \quad t \in[a, b], \\
u(\tau)=0,
\end{gathered}
$$

obviously, coincides with that of the equation

$$
u(t)=\int_{\tau}^{t} p(s) u(\omega(s)) d s, \quad t \in[a, b] .
$$

The linear operator

$$
C([a, b], \mathbb{R}) \ni u \longmapsto \int_{\tau} p(s) u(\omega(s)) d s,
$$

as is easy to show, is a compact self-mapping of the space $C([a, b], \mathbb{R})$ of continuous functions on the interval $[a, b]$, whenever $p:[a, b] \rightarrow \mathbb{R}$ is integrable and $\omega:[a, b] \rightarrow[a, b]$ is measurable.

The paper is organised as follows. Section 2 contains some definitions and preliminary results. In Section 3, we establish an estimate for the so-called $K$-substantial eigenvalues (see Definition 2.44) of a bounded linear operator in a Banach space with a wedge $K$. The main Theorem 4.1 of Section 4 provides a convenient upper bound for the spectral radius of a completely continuous linear operator vanishing on the blade of the wedge $K$ containing elements that are strongly positive in the sense of Definition 2.12. Finally, in the last Section 7, Theorem 4.1 is applied to obtain conditions sufficient for the solvability of certain integro-functional equations.

\section{WEDGES IN BANACH SPACES AND LINEAR OPERATORS LEAVING THEM INVARIANT}

In this section, we recall the basic definitions related to wedges in Banach spaces, introduce some notation and definitions, and establish a number of statements relied upon in the subsequent sections. Throughout the rest of this paper, $X$ is a Banach space over the field $\mathbb{R}$.

2.1. Wedges and related preorderings. A closed subset $K$ of $X$ is said to be a wedge (see, e. g., [6]) if

$$
\alpha_{1} K+\alpha_{2} K \subseteq K
$$

for all $\left\{\alpha_{1}, \alpha_{2}\right\} \subset[0,+\infty)$, where, as usual, $\alpha_{1} K+\alpha_{2} K:=\left\{\alpha_{1} x_{1}+\alpha_{2} x_{2} \mid\left\{x_{1}, x_{2}\right\} \subset K\right\}$. 
In what follows, we assume implicitly that the wedge $K$ is proper, i. e., is different from both the singleton $\{0\}$ and the entire space $X$, for there is no meaningful theory in those two extreme cases.

Remark 2.1. In the original terminology introduced by M. Krein [10], a closed set satisfying condition (2.1) is called a linear semigroup.

The following standard definition introduces a natural preordering in a space $X$ with a wedge $K$.

Definition 2.2. The relation $x_{1} \leqq_{K} x_{2}$ is said to be satisfied if, and only if $x_{2}-x_{1} \in$ $K$.

We also write $x_{1} \geqq_{K} x_{2}$ if, and only if $x_{2} \leqq_{K} x_{1}$. Note that the relations $x_{1} \leqq_{K} x_{2}$ and $x_{1} \geqq_{K} x_{2}$, generally speaking, do not imply the equality $x_{1}=x_{2}$.

Definition 2.3. The set $K \cap(-K)$ is referred to as the blade [6] of the wedge $K$.

For the sake of brevity, we shall denote the blade of the wedge $K$ by the symbol $K^{\diamond}$ :

$$
K^{\diamond}:=\left\{x \in X \mid x \geqq_{K} 0 \wedge x \varliminf_{K} 0\right\} .
$$

Remark 2.4. It is obvious from condition (2.1) and definition (2.2) that the blade of an arbitrary wedge $K$ is a closed linear subset of $K$. One can readily show that $K^{\diamond}$ coincides with the maximal linear subspace contained in $K$.

Definition 2.5. We write $x_{1} \square_{K} x_{2}$ if, and only if either $x_{1} \geqq_{K} x_{2}$ or $x_{1} \geqq_{K} x_{2}$.

The relation $\square_{K}$ is obviously reflexive and symmetric.

2.2. Measurable elements of a Banach space. Let $f$ be an element from $X, \beta$ be a real constant, and $X_{K, \beta}(f)$ be the set defined as follows:

$$
X_{K, \beta}(f):=\left\{x \in X \mid-\beta f \leqq_{K} x \leqq_{K} \beta f\right\} .
$$

2.2.1. Basic properties of the sets $X_{K, \beta}(f)$. In the sequel, we need some properties of sets (2.3).

Lemma 2.6. Let $\beta$ be a fixed real number. Then an element $x$ from $X$ belongs to the set $X_{K, \beta}(f)$ if, and only if $-x \in X_{K, \beta}(f)$.

Proof. Due to the symmetry of the left-hand and right-hand terms, the inequality

$$
-\beta f \leqq_{K} x \leqq_{K} \beta f
$$

is equivalent to the relation

$$
-\beta f \leqq_{K}-x \leqq_{K} \beta f,
$$

whereas the latter means that $-x \in X_{K, \beta}(f)$.

Lemma 2.7. The following assertions are true:

(i) $X_{K, 0}(f)=K^{\diamond}$ for all $f \in X$; 
(ii) $X_{K, \beta}(0)=K^{\diamond}$ for any $\beta \in \mathbb{R}$;

(iii) The set $X_{K, \beta}(f)$, where $\beta \neq 0$ and $f \neq 0$, is non-empty if, and only if $\beta f \geqq_{K} 0$;

(iv) If $\beta f \geqq_{K} 0$, then $X_{K, \beta}(f) \supseteq K^{\diamond}$;

(v) If $f \in K^{\diamond}$, then $X_{K, \beta}(f)=K^{\diamond}$ for all $\beta \in \mathbb{R}$;

(vi) $X_{K, \beta}(f) \backslash K^{\diamond} \neq \varnothing$ if, and only if $\beta f \geqq_{K} 0$ and $\beta f \notin K^{\diamond}$.

Proof. Assertions (i) and (ii) are obvious from (2.3). Let us verify assertion (iii). Indeed, let $x$ belong to $X_{K, \beta}(f)$. This is true if, and only if (2.4) holds or, which is the same (see Lemma 2.6), relation (2.5) is satisfied. Combining (2.4) and (2.5) and using property (2.1) of $K$, we obtain

$$
-2 \beta f \varliminf_{K} 0 \varliminf_{K} 2 \beta f,
$$

i. e., $\beta f \geqq_{K} 0$. Conversely, if $\beta f \geqq_{K} 0$, then, in particular,

$$
-\beta f \leqq_{K} \beta f \leqq_{K} \beta f .
$$

This means that (2.4) is satisfied with $x=\beta f$, i. e., $\beta f \in X_{K, \beta}(f)$.

To prove assertion (iv), it is sufficient to note that if $\beta f \geqq_{K} 0$, then (2.4) is true for all elements $x$ satisfying the relation $0 \leqq_{K} x \leqq_{K} 0$.

Let $f \in K^{\diamond}$ be arbitrary. By (iv), we have $K^{\diamond} \subset X_{K, \beta}(f)$ for all $\beta \in \mathbb{R}$. On the other hand, if $x \in X_{K, \beta}(f)$, then, according to (2.4), we obtain $x \in K^{\diamond}$ because $\beta f$ is also an element of $K^{\diamond}$. Thus, assertion (v) is true.

Finally, assertion (vi) is obvious from (iii), (iv), and (v).

Assertions (i) and (ii) of Lemma 2.7 show that there is no much sense to consider the sets $X_{K, \beta}(f)$ with $\beta f=0$ because, in that case, they consist solely of those elements of $X$ which are 0-measurable with respect to $K$ in the sense of Definition 2.8 given below.

2.2.2. The definition of $f$-measurability. For the sake of brevity, we shall use the following

Definition 2.8. An element $x$ from $X$ is said to be $f$-measurable with respect to $K$ if there exists a real constant $\beta$ such that $x \in X_{K, \beta}(f)$.

In other words, $x$ is $f$-measurable with respect to the wedge $K$ whenever (2.4) holds for some $\beta$.

Remark 2.9. Definition 2.8 differs from a similar notion introduced in [10] because the negative values of $\beta$ are allowed in (2.4). For the purposes of this paper, the definition mentioned seems to be advantageous due to the need to consider complexifications (see Section 2.5 below). Note also that, according to Definition 2.8, the set of $f$-measurable elements is never empty (see Proposition 2.11).

Definition 2.10. For every fixed $f \in X$, the set of all the elements of $X$ that are $f$-measurable with respect to $K$ will be denoted by $X_{K}(f)$. 

fact,

Clearly, $X_{K}(f):=\bigcup_{\beta \in \mathbb{R}} X_{K, \beta}(f)$. Moreover, it follows from Lemma 2.7 that, in

$$
X_{K}(f)=\bigcup_{\beta \in \mathbb{R}: \beta f \geqq_{K} 0} X_{K, \beta}(f)
$$

for any $f \in X$.

Proposition 2.11. For any $f \in X$, the set $X_{K}(f)$ is a linear manifold containing $K^{\diamond}$. Furthermore, $X_{K}(f) \neq K^{\diamond}$ if, and only if the element $f$ is such that $f \square_{K} 0$ and $f \notin K^{\diamond}$.

Proof. The set $X_{K}(f)$ obviously satisfies the condition

$$
\alpha_{1} X_{K}(f)+\alpha_{2} X_{K}(f) \subset X_{K}(f)
$$

for all $\left\{\alpha_{1}, \alpha_{2}\right\} \subset[0,+\infty)$ and, therefore, Lemma 2.6 guarantees that it is a linear manifold.

According to Definition 2.10 and assertion (i) of Lemma 2.7, we have $X_{K}(f)$ ᄀ $X_{K, 0}(f)=K^{\diamond}$. Furthermore, equality (2.6) yields

$$
X_{K}(f) \backslash K^{\diamond}=\bigcup_{\beta \in \mathbb{R}: 0 \neq \beta f \geqq_{K} 0} X_{K, \beta}(f) \backslash K^{\diamond} .
$$

However, assertion (vi) of Lemma 2.7 guarantees that the condition $f \notin K^{\diamond}$ is necessary and sufficient for the union in the right-hand side of (2.7) to contain non-empty sets.

2.3. Strict inequalities with respect to a wedge. Given a wedge $K \subseteq X$ and a linear manifold $H$ in $X$, we introduce the following binary relation on $X$.

Definition 2.12. For $\left\{f_{1}, f_{2}\right\} \subset X$, we write $f_{1} \varpi_{K ; H} f_{2}$ if, and only if the inclusion

$$
X_{K}\left(f_{2}-f_{1}\right) \supseteq H
$$

is satisfied.

One can readily verify that the equality

$$
X_{K}(-f)=X_{K}(f)
$$

holds for any $f$ and, hence, the relation introduced by Definition 2.12 is symmetric, i. e., $f_{1} \varpi_{K ; H} f_{2}$ if, and only if $f_{2} \varpi_{K ; H} f_{1}$.

Lemma 2.13. If $f_{1} \varpi_{K ; H_{1}} f_{2}$ and $H_{1} \supseteq H_{2}$, then $f_{1} \varpi_{K ; H_{2}} f_{2}$.

The assertion of the last lemma is easily established by using Definition 2.12.

Lemma 2.14. For an arbitrary $f$ from $X$, the relation

$$
f \varpi_{K ; X_{K}(f)} 0
$$

is true. 
Proof. By Proposition 2.11, the set $X_{K}(f)$ is a linear manifold in $X$. According to Definition 2.12, relation (2.8) is equivalent to the inclusion $X_{K}(f) \supseteq X_{K}(f)$ and, hence, is always satisfied.

In the case where $H=X$, we drop the corresponding subscript in the above notation and, instead of $f_{1} \varpi_{K ; X} f_{2}$, we write $f_{1} \varpi_{K} f_{2}$ :

Definition 2.15. For $\left\{f_{1}, f_{2}\right\} \subset X$, we write $f_{1} \varpi_{K} f_{2}$ if, and only if $X_{K}\left(f_{2}-f_{1}\right)=X$.

The above definition allows one to introduce the following

Definition 2.16. Two elements $f_{1}$ and $f_{2}$ are said to be in the relation $f_{1} \varepsilon_{K ; H} f_{2}$ (resp., $f_{1}-3_{K} ; H f_{2}$ ) if they satisfy the conditions $f_{1} \varpi_{K ; H} f_{2}$ and $f_{1} \geqq_{K} f_{2}$ (resp., $\left.f_{1} \leqq_{K} f_{2}\right)$.

By analogy with Definition 2.15, we introduce

Definition 2.17. Two elements $f_{1}$ and $f_{2}$ are said to be in the relation $f_{1} \varepsilon_{K} f_{2}$ (resp., $f_{1}-_{K} f_{2}$ ) if they satisfy the conditions $f_{1} \square_{K} f_{2}$ and $f_{1} \geqq_{K} f_{2}$ (resp., $f_{1} \leqq_{K} f_{2}$ ).

The fulfilment of the relations described by Definition 2.17 is verified most easily in the case of a solid wedge.

Definition 2.18. A wedge is said to be solid [10] if its interior is non-empty.

Following [10], we write $x_{1} \ll_{K} x_{2}$ (resp., $x_{1} \gg_{K} x_{2}$ ) if, and only if the difference $x_{2}-x_{1}$ (resp., $\left.x_{1}-x_{2}\right)$ lies in the interior of $K$.

Lemma 2.19. If $K$ is a solid wedge in $X$ and an element $f \in X$ is such that $f \gg_{K} 0$, then $f$ satisfies the relation

$$
f \varepsilon_{K} 0 .
$$

Proof. A statement equivalent to equality (2.14) for $f$ lying in the interior of $K$ is well-known, e. g., from $[10,14]$.

Remark 2.20. When $K$ is a minihedral cone in $X$ [10] (and, hence, the partial ordering $\leqq_{K}$ makes $X$ into a vector lattice [1]), an element $u$ possessing the property $u \varepsilon_{K} 0$ is called a strong unit (see Definition XIII.1.5 in [14]). In this case, the condition $X_{K}(u)=X$ means that the element $u$ satisfies Axiom $\mathrm{V}$ from [9].

Remark 2.21. Relation (2.9), generally speaking, does not imply that $f \gg_{K} 0$. For example, in the space $L_{\infty}([0,1])$ of essentially bounded functions endowed with the usual norm and partial ordering [14], relation (2.9) is true, e. g., for $f$ equal almost everywhere to 1 . However, the set of functions non-negative almost everywhere on $[0,1]$ has empty interior in $L_{\infty}([0,1])$.

For suitable linear manifolds $H$, the condition

$$
f \varepsilon_{K ; H} 0
$$


may be regarded as a certain "strong positivity" an element $f \geqq_{K} 0$. The word "suitable" here means that, roughly speaking, there should not be too many strongly positive elements. For instance, there is no much sense to study the case where

$$
H \subseteq K^{\diamond}
$$

because, by virtue of Proposition 2.11, the inclusion

$$
\bigcap_{f \in X} X_{K}(f) \supseteq K^{\diamond}
$$

is always true and, hence, under condition (2.10), the relation $f \varepsilon_{K ; K^{\diamond}} 0$ is satisfied by an arbitrary element $f$ from $X$. On the other hand, certain undesirable classes of vectors $f$ (e. g., $f=0$ or, more generally, $f$ satisfying the relation $0 \leqq_{K} f \leqq_{K} 0$ ), that are unlikely candidates for strongly positive elements, should also be excluded from consideration. These considerations lead us to the following

Proposition 2.22. Let $H$ be a linear manifold in $X$ such that

$$
H \nsubseteq K^{\diamond}
$$

and $f$ be an element of $X$ such that either the relation $f \square_{K} 0$ is not true or $0 \leqq K$ $f \leqq_{K} 0$. Then the relation

$$
f \varpi_{K ; H} 0
$$

is not satisfied.

Proof. Indeed, let, on the contrary, relation (2.12) holds. According to Definition 2.12, this means that $H \subseteq X_{K}(f)$ and, therefore, in view of condition (2.11), the set $X_{K}(f)$ contains some elements not belonging to $K^{\diamond}$. It then follows from Proposition 2.11 that $f$ should satisfy the relations $f \square_{K} 0$ and $f \notin K^{\diamond}$, contrary to the assumption.

In other words, Proposition 2.22 means that a strongly positive element should always be comparable with zero and cannot be positive and negative simultaneously. This agrees well with the intuitive idea of the strict inequality.

2.4. The mappings $n_{K, f}: X_{K}(f) \rightarrow[0,+\infty)$. Taking a glance at Definition 2.10, we see that the non-negative number

$$
n_{K, f}(x):=\inf \left\{|\beta| \mid \beta \in(-\infty,+\infty) \text { and } x \in X_{K, \beta}(f)\right\}
$$

is well-defined for an arbitrary $x$ from $X_{K}(f)$. It is also convenient to put $n_{K, f}(x):=$ $+\infty$ for all $x \in X \backslash X_{K}(f)$. Thus, $n_{K, f}(x)<+\infty$ if, and only if $x$ is $f$-measurable with respect to $K$.

Remark 2.23. One can show that, for any $f \in X$, the mapping $n_{K, f}: X_{K}(f) \rightarrow$ $[0,+\infty)$ is a seminorm on the linear manifold $X_{K}(f)$. This seminorm is a norm if, and 
only if $K$ is a cone [5,10], i. e., if the blade of $K$ is trivial. The mapping mentioned is defined on the entire space $X$ if, and only if

$$
X_{K}(f)=X,
$$

which property, in contrast to the poorest case where

$$
X_{K}(f)=K^{\diamond},
$$

may be regarded as a reflection of a reasonable choice of an element $f \geqq_{K} 0$. By Lemma 2.19, condition (2.14) is satisfied if $f \gg_{K} 0$. It may happen, however, that (2.14) does not hold for any $f$ from $X$ (e. g., if $X$ is the Banach space of the Lebesgue integrable functions on a bounded interval $[a, b]$ and $K$ is the cone of integrable functions $[a, b] \rightarrow \mathbb{R}$ that are non-negative almost everywhere on $[a, b])$.

In the case where $K$ is a solid cone and $f \gg_{K} 0$, formula (2.13) determines the so-called $f$-norm $[7,10]$

$$
\|x\|_{f}=\inf \{\beta \in[0,+\infty) \mid \text { relation (2.4) is true }\}
$$

of an arbitrary element $x$ from $X$. Functional (2.15) is also used in [9] in studies of vector lattices.

It is clear from (2.13) that $n_{K, f}(0)=0$ independently of the choice of $f$. Moreover, the following lemma holds.

Lemma 2.24. Let $f \in X$. Then an element $x \in X$ satisfies the equality

$$
n_{K, f}(x)=0
$$

if, and only if $x \in K^{\diamond}$.

Proof. Let $f \in X$ and let $x$ be an element from the corresponding (non-empty) set $X_{K}(f)$. In view of Proposition 2.11, we can suppose that $f \square_{K} 0$. Then, clearly, $\sigma f \geqq_{K} 0$ for some $\sigma \in\{-1,1\}$.

Let $x \in K^{\diamond}$. The element $x$ belongs to the blade of $K$ if, and only if

$$
0 \leqq_{K} x \leqq_{K} 0 \text {, }
$$

which means that (2.4) is true with an arbitrary constant $\beta$ such that $\operatorname{sign} \beta=\sigma$. In particular,

$$
-\frac{\sigma}{k} f \varliminf_{K} x \leqq_{K} \frac{\sigma}{k} f
$$

for all $k \in \mathbb{N}$. Taking (2.13) into account, we conclude that $0 \leq n_{K, f}(x) \leq \inf _{k \in \mathbb{N}} k^{-1}=$ 0 , i. e., relation (2.16) holds.

Conversely, if $x$ satisfies equality (2.16), then there exists a sequence $\left(\beta_{k}\right)_{k=1}^{+\infty} \subset$ $(-\infty,+\infty)$ such that $\lim _{k \rightarrow+\infty} \beta_{k}=0$ and, for all $k \geq 1$,

$$
-\beta_{k} f \leqq_{K} x \leqq_{K} \beta_{k} f \text {. }
$$

Passing to the limit as $k \rightarrow+\infty$ in relation (2.18) or, which is the same, in the inclusion

$$
\left\{\beta_{k} f-x, \beta_{k} f+x\right\} \subset K
$$


and taking into account the fact that $K$ is a closed set, we arrive at relation (2.17).

Remark 2.25. The existence of an element $f$ satisfying equality (2.14) implies, in particular, that the wedge $K$ is reproducing [10], i. e., $K-K=X$. The converse implication, generally speaking, is not true (in particular, the cone mentioned in Remark 7.4 is reproducing but relation (2.14) is never satisfied there).

2.5. Complexification of a wedge and the related objects. In the sequel, the complex counterparts of some of the notions defined above will be needed. Throughout this section, where the related notions are introduced, we fix a real Banach space $X$ and wedge $K$ in $X$.

2.5.1. Basic issues. The complexification (see, e. g., [4], Chapt. XIII, §2) of a real Banach space $\langle X,\|\cdot\|\rangle$ is convenient to be interpreted as the complex Banach space $\hat{X}$ of formal sums $x+\mathrm{i} y,\{x, y\} \subset X, \mathrm{i}^{2}=-1$, equipped with the linear operations

$$
\begin{gathered}
\left(x_{1}+\mathrm{i} y_{1}\right)+\left(x_{2}+\mathrm{i} y_{2}\right):=x_{1}+x_{2}+\mathrm{i}\left(y_{1}+y_{2}\right), \\
(v+\mathrm{i} \mu)(x+\mathrm{i} y):=v x-\mu y+\mathrm{i}(\mu x+v y),
\end{gathered}
$$

where $\left\{x_{1}, x_{2}, y_{1}, y_{2}, x, y\right\} \subset X,\{v, \mu\} \subset \mathbb{R}$, and with the norm

$$
\|x+\mathrm{i} y\|:=\max _{\vartheta \in[-\pi, \pi]}\|x \cos \vartheta+y \sin \vartheta\|, \quad\{x, y\} \subset X .
$$

The same technique allows one to define a natural complexification of an arbitrary wedge in a real Banach space.

Definition 2.26. The set

$$
\hat{K}:=\{x+\mathrm{i} y \mid x \in K \wedge y \in K\}
$$

will be referred to as the complexification of a wedge $K$ in a Banach space $X$ over $\mathbb{R}$.

It is easy to verify that the set $\hat{K}$, represented alternatively as $\hat{K}=K+\mathrm{i} K$, is closed with respect to norm (2.20) and forms a wedge in $\hat{X}$ in the sense that

$$
\alpha_{1} \hat{K}+\alpha_{2} \hat{K} \subset \hat{K} \quad \text { for all }\left\{\alpha_{1}, \alpha_{2}\right\} \subset[0,+\infty) .
$$

By analogy with Sections 2.1 and 2.3, one can extend the binary relations $\leqq_{K}$ and $\ll_{K}$ to $\hat{X}^{2}$ in a natural way. More precisely, given two elements $\left\{z_{1}, z_{2}\right\} \subset \hat{X}$, we shall write $z_{1} \geqq_{\hat{K}} z_{2}$ (resp., $z_{1} \gg_{\hat{K}} z_{2}$ ) if, and only if $z_{1}-z_{2} \in \hat{K}$ (resp., $z_{1}-z_{2}$ is an interior element of $\hat{K}$ ). Similarly, the relation $\square_{\hat{K}}$ is natural to be defined by putting $z_{1} \square_{\hat{K}} z_{2}$ if, and only if the elements $z_{1}$ and $z_{2}$ satisfy at least one of the relations $z_{1} \geqq_{\hat{K}} z_{2}$ and $z_{1} \leqq_{\hat{K}} z_{2}$. The blade $(\hat{K})^{\diamond}$ of $\hat{K}$ is natural to be defined as the set of all those $z$ from $\hat{X}$ for which both relations $z \geqq_{\hat{K}} 0$ and $z \leqq_{\hat{K}} 0$ are true, i. e.,

$$
(\hat{K})^{\diamond}=\hat{K} \cap(-\hat{K}) \text {. }
$$

It is obvious that

$$
(\hat{K})^{\diamond}=K^{\diamond}+\mathrm{i} K^{\diamond}
$$


The complexification $\hat{K}$ of a real wedge $K$ inherits its main characteristic properties. For example, $\hat{K}$ is solid if, and only if $K$ possesses this property.

2.5.2. Measurability of complex elements. Let $g \in \hat{X}$ and $\lambda \in \mathbb{C}$. Similarly to formula (2.3), one can define the set $\hat{X}_{\hat{K}, \lambda}(g) \subset \hat{X}$ by putting

$$
\hat{X}_{\hat{K}, \lambda}(g):=\left\{z \in \hat{X} \mid z \geqq_{\hat{K}}-\lambda g \wedge z \leqq_{\hat{K}} \lambda g\right\}
$$

and introduce the following

Definition 2.27. An element $z \in \hat{X}$ is said to be g-measurable with respect to the wedge $\hat{K}$ if, and only if it belongs to the set

$$
\hat{X}_{\hat{K}}(g):=\bigcup_{\lambda \in \mathbb{C}} \hat{X}_{\hat{K}, \lambda}(g) .
$$

Definition 2.27 may be regarded as a natural extension of Definition 2.8 to the complex case. For example, analogues of Lemma 2.6 and Proposition 2.11 are true for sets (2.24) and, just as in the real case, zero belongs to the set $\hat{X}_{\hat{K}, \lambda}(g)$ for arbitrary $\lambda \in \mathbb{C}$ and $g \in \hat{X}$. Further properties of sets (2.24) are described by Lemma 2.33 below.

Remark 2.28. Analogues of sets (2.24) and the related objects can also be introduced in the case where $\hat{K}$ is replaced by some other set possessing property (2.22), not necessarily constructed according to formula (2.21). Such more general complex wedges are however not needed for our purposes.

A convenient characterisation of the property introduced in Definition 2.27 is provided by the following

Lemma 2.29. Let $\{x, y, f\} \subset X$. Then the element $x+\mathrm{i} y$ is $\hat{f}$-measurable with respect to $\hat{K}$ if, and only if there exist some $r \in[0,+\infty)$ and $\omega \in[-\pi, \pi]$ such that the relations

$$
\begin{aligned}
& -r f \sin \omega \leqq_{K} x \leqq_{K} r f \sin \omega, \\
& -r f \cos \omega \leqq_{K} y \leqq_{K} r f \cos \omega
\end{aligned}
$$

are satisfied.

Here and everywhere in the sequel, we write $\hat{f}=f+$ if $f$ for any $f$ from $X$.

Proof. By virtue of relations (2.24) and (2.25), the element $x+\mathrm{i} y$ is $\hat{f}$-measurable if, and only if there exist some $\varrho \in[0,+\infty)$ and $\vartheta \in[-\pi, \pi]$ for which

$$
-\varrho \mathrm{e}^{\mathrm{i} \vartheta} \hat{f} \leqq_{\hat{K}} x+\mathrm{i} y \leqq_{\hat{K}} \varrho \mathrm{e}^{\mathrm{i} \vartheta} \hat{f} .
$$

According to (2.19), we have

$$
\begin{aligned}
\mathrm{e}^{\mathrm{i} \vartheta} \hat{f} & =(\cos \vartheta+\mathrm{i} \sin \vartheta)(f+\mathrm{i} f)=(\cos \vartheta-\sin \vartheta) f+\mathrm{i}(\sin \vartheta+\cos \vartheta) f \\
& =\sqrt{2}\left[\sin \left(\frac{\pi}{4}-\vartheta\right)+\mathrm{i} \cos \left(\frac{\pi}{4}-\vartheta\right)\right] f .
\end{aligned}
$$


Therefore, in view of definition (2.21) of the set $\hat{K}$, the relation (2.28) is equivalent to the system of order inequalities

$$
\begin{aligned}
& -\varrho \sqrt{2} f \sin \left(\frac{\pi}{4}-\vartheta\right) \leqq_{K} x \leqq_{K} \varrho \sqrt{2} f \sin \left(\frac{\pi}{4}-\vartheta\right), \\
& -\varrho \sqrt{2} f \cos \left(\frac{\pi}{4}-\vartheta\right) \leqq_{K} y \leqq_{K} \varrho \sqrt{2} f \cos \left(\frac{\pi}{4}-\vartheta\right),
\end{aligned}
$$

which, obviously, has form (2.26), (2.27) with $r:=\varrho \sqrt{2}$ and

$$
\omega:= \begin{cases}\frac{\pi}{4}-\vartheta & \text { if }-\pi \leq \vartheta \leq-\frac{3 \pi}{4}, \\ -\frac{7 \pi}{4}-\vartheta & \text { if }-\frac{3 \pi}{4}<\vartheta \leq \pi .\end{cases}
$$

It is clear that the above relation between the pairs $(\varrho, \vartheta)$ and $(r, \omega)$ is one-to-one.

Remark 2.30. Definition 2.27 reduces to Definition 2.8 in the real case. Indeed, let $\sigma f \in \hat{K}$ with some $\sigma \in\{-1,1\}$. Lemma 2.29 characterises the $\hat{f}$-measurability of the element $x=x+\mathrm{i} 0$ with respect to $\hat{K}$ in terms of the existence of $(r, \omega) \in$ $[0,+\infty) \times[-\pi, \pi]$ such that $\sigma \cos \omega \geq 0$ and relation (2.26) is true. However, the property mentioned means that (2.4) is satisfied with $\beta:=r \sin \omega$.

It is natural to find out that the $\hat{f}$-measurability of an element $x+\mathrm{i} y$ with respect to $\hat{K}$ is equivalent to the $f$-measurability of its real and imaginary parts, $x$ and $y$.

Lemma 2.31. Let $\{x, y, f\} \subset X$. Then the element $x+\mathrm{i} y$ is $\hat{f}$-measurable with respect to $\hat{K}$ if, and only if both $x$ and $y$ are $f$-measurable with respect to $K$.

Proof. The $f$-measurability of $x$ and $y$, on the assumption that $x+\mathrm{i} y \in \hat{X}_{\hat{K}}(\hat{f})$, is a consequence of Lemma 2.29. Conversely, if $\{x, y\} \subset X_{K}(f)$, then, according to Definition 2.8, there exist some real $\alpha$ and $\beta$ such that

$$
\begin{aligned}
& -\alpha f \leqq_{K} x \leqq_{K} \alpha f, \\
& -\beta f \leqq_{K} y \leqq_{K} \beta f .
\end{aligned}
$$

Let us put

$$
\omega:= \begin{cases}\frac{\pi}{4} \operatorname{sign} \alpha & \text { if } \beta \geq 0, \\ \frac{3 \pi}{4} \operatorname{sign} \alpha & \text { if } \beta<0\end{cases}
$$

and $r:=\sqrt{2} \max \{|\alpha|,|\beta|\}$. Then, as is easy to see,

$$
\sin \omega=\frac{\operatorname{sign} \alpha}{\sqrt{2}}, \quad \cos \omega=\frac{\operatorname{sign} \beta}{\sqrt{2}},
$$

and, therefore, relations (2.32) and (2.33) imply that (2.26) and (2.27) are satisfied with the above values of $\omega$ and $r$. It remains to refer to Lemma 2.29.

It turns out that all the sets $\hat{X}_{\hat{K}}(\hat{f})$, where $\hat{f}=f+\mathrm{i} f$, are invariant under rotations. More precisely, the following statement is true. 
Lemma 2.32. Let $f \in X$ and $\{x, y\} \subset X_{K}(f)$. Then, for an arbitrary $\varphi \in[-\pi, \pi]$, the element $\mathrm{e}^{\mathrm{i} \varphi}(x+\mathrm{i} y)$ is $\hat{f}$-measurable with respect to $\hat{K}$.

Proof. It will suffice to consider the case where $f \square_{K} 0$. By assumption, $\{x, y\} \subset$ $X_{K}(f)$ and, hence, in view of Lemma 2.31, the element $x+\mathrm{i} y$ is $\hat{f}$-measurable with respect to $\hat{K}$, where $\hat{f}=f+\mathrm{i} f$. Lemma 2.29 guarantees the existence of an $\omega \in[-\pi, \pi]$ such that relations (2.26) and (2.27) are satisfied. Multiplying both parts of (2.26) by $|\cos \varphi|$ and $|\sin \varphi|$ and taking Lemma 2.6 into account, we obtain, respectively, the relations

$$
-f r|\cos \varphi| \sin \omega \leqq_{K} \pm x \cos \varphi \leqq_{K} f r|\cos \varphi| \sin \omega
$$

and

$$
-f r|\sin \varphi| \sin \omega \varliminf_{K} \pm x \sin \varphi \leqq_{K} f r|\sin \varphi| \sin \omega,
$$

where the symbol " \pm " means that the inequality is satisfied with both signs of the corresponding term. Similarly, multiplying both parts of (2.27) by $|\cos \varphi|$ and $|\sin \varphi|$, we get

$$
-f r|\cos \varphi| \cos \omega \leqq_{K} \pm y \cos \varphi \leqq_{K} f r|\cos \varphi| \cos \omega
$$

and

$$
-f r|\sin \varphi| \cos \omega \leqq_{K} \pm y \sin \varphi \leqq_{K} f r|\sin \varphi| \cos \omega .
$$

Therefore, by choosing the appropriate signs in the relations above and summing the corresponding terms, we obtain

$$
\begin{aligned}
& x \cos \varphi-y \sin \varphi \leqq_{K} r f[|\cos \varphi| \sin \omega+|\sin \varphi| \cos \omega], \\
& x \cos \varphi-y \sin \varphi \geqq_{K}-r f[|\cos \varphi| \sin \omega+|\sin \varphi| \cos \omega]
\end{aligned}
$$

and

$$
\begin{aligned}
& y \cos \varphi+x \sin \varphi \leqq_{K} r f[|\cos \varphi| \cos \omega+|\sin \varphi| \sin \omega], \\
& y \cos \varphi+x \sin \varphi \geqq_{K}-r f[|\cos \varphi| \cos \omega+|\sin \varphi| \sin \omega] .
\end{aligned}
$$

It is supposed that $f \square_{K} 0$, and, therefore, $\sigma f \geqq_{K} 0$ for some $\sigma \in\{-1,1\}$. Since neither $|\cos \varphi| \sin \omega+|\sin \varphi| \cos \omega$ nor $|\cos \varphi| \cos \omega+|\sin \varphi| \sin \omega$ takes values outside the interval $[-2,2]$, relations (2.34) and (2.35) yield

$$
\begin{aligned}
& 2 \sigma r f \leqq_{K} x \cos \varphi-y \sin \varphi \leqq_{K} 2 \sigma r f, \\
& 2 \sigma r f \leqq_{K} y \cos \varphi+x \sin \varphi \leqq_{K} 2 \sigma r f .
\end{aligned}
$$

Let us put

$$
\vartheta_{\sigma}:= \begin{cases}\frac{\pi}{4} & \text { if } \sigma=1, \\ \frac{-3 \pi}{4} & \text { if } \sigma=-1 .\end{cases}
$$

Then $\sin \vartheta_{\sigma}=\cos \vartheta_{\sigma}=\sigma 2^{-\frac{1}{2}}$ and, therefore, relations (2.36) and (2.37) can be brought to the form

$$
\begin{aligned}
& \varrho f \sin \vartheta_{\sigma} \leqq_{K} x \cos \varphi-y \sin \varphi \leqq_{K} \varrho f \sin \vartheta_{\sigma}, \\
& \varrho f \cos \vartheta_{\sigma} \leqq_{K} y \cos \varphi+x \sin \varphi \leqq_{K} \varrho f \cos \vartheta_{\sigma},
\end{aligned}
$$


where $\varrho:=2 r \sqrt{2}$. Applying now Lemma 2.29 and taking into account the formula

$$
\mathrm{e}^{\mathrm{i} \varphi}(x+\mathrm{i} y)=x \cos \varphi-y \sin \varphi+\mathrm{i}(y \cos \varphi+x \sin \varphi),
$$

we conclude that the element $\mathrm{e}^{\mathrm{i} \varphi}(x+\mathrm{i} y)$ is $\hat{f}$-measurable.

The next lemma summarises several properties of sets (2.24) referred to in the sequel.

Lemma 2.33. The following assertions are true:

(i) $\hat{X}_{\hat{K}, 0}(\hat{f})=\hat{X}_{\hat{K}, \lambda}(0)=K^{\diamond}+\mathrm{i} K^{\diamond}$ for all $f \in X$ and $\lambda \in \mathbb{C}$;

(ii) $\hat{X}_{\hat{K}, \lambda}(g)=-\hat{X}_{\hat{K}, \lambda}(g)$ for any $g \in \hat{X}$;

(iii) $\alpha^{-1} \hat{X}_{\hat{K},|\alpha| \lambda}(g)=\hat{X}_{\hat{K}, \lambda}(g)$ for any $g \in \hat{X}$ and $\alpha \in \mathbb{R} \backslash\{0\}$;

(iv) For $\lambda \neq 0$ and $g \neq 0$, the set $\hat{X}_{\hat{K}, \lambda}(g)$ is non-empty if, and only if $\lambda g \in \hat{K}$;

(v) If $\lambda g \in \hat{K}$, then $\hat{X}_{\hat{K}, \lambda}(g) \supseteq K^{\diamond}+\mathrm{i} K^{\diamond}$;

(vi) $\hat{X}_{\hat{K}, \lambda}(g) \backslash\left(K^{\diamond}+\mathrm{i} K^{\diamond}\right) \neq \varnothing$ if, and only if $\lambda g \in \hat{K} \backslash\left(K^{\diamond}+\mathrm{i} K^{\diamond}\right)$.

(vii) $\bigcap_{g \in \hat{X}} \hat{X}_{\hat{K}}(g) \supseteq K^{\diamond}+\mathrm{i} K^{\diamond}$;

(viii) $\hat{X}_{\hat{K}}(g) \neq K^{\diamond}+\mathrm{i} K^{\diamond}$ if, and only if $g \in[\hat{K} \cup(-\hat{K})] \backslash\left(K^{\diamond}+\mathrm{i} K^{\diamond}\right)$.

Proof. This statement is established similarly to Lemmata 2.6 and 2.7 and Propositions 2.11 and 2.11 from Section 2.2.

Let us prove, e. g., assertion (iii). Indeed, let $\alpha \neq 0$. By virtue of (ii), an element $z$ belongs to the set $\hat{X}_{\hat{K},|\alpha| \lambda}$ if, and only if

$$
-|\alpha| \lambda g \leqq_{\hat{K}} z \operatorname{sign} \alpha \leqq_{\hat{K}}|\alpha| \lambda g,
$$

or, which is the same,

$$
-\lambda g \leqq_{\hat{K}} \frac{z}{\alpha} \leqq_{\hat{K}} \lambda g .
$$

However, (2.39) means nothing but the inclusion $\alpha^{-1} z \in \hat{X}_{\hat{K}, \lambda}(g)$.

2.5.3. The mappings $\boldsymbol{n}_{\hat{\boldsymbol{K}}, \boldsymbol{g}}: \hat{\boldsymbol{X}} \rightarrow[\mathbf{0},+\infty]$. Similarly to the case of the original real space $X$, the $\hat{f}$-measurability of elements of $\hat{X}$ with respect to the complexification $\hat{K}$ of a wedge $K$ in $X$ can be characterised by a certain non-linear functional. More precisely, given $z \in \hat{X}$ and $g \in \hat{X}$, we put

$$
n_{\hat{K}, g}(z):=\sqrt{2} \inf \left\{|\lambda| \mid \lambda \in \mathbb{C} \wedge z \in \hat{X}_{\hat{K}, \lambda}(g)\right\}
$$

if $z$ is $g$-measurable with respect to $\hat{K}$, and $n_{\hat{K}, g}(z):=+\infty$ for $z \notin \hat{X}_{\hat{K}}(g)$. Here, we retain the same letter, $n$, as in the real case (cf. Section 2.2) in order not to complicate the notation unnecessarily.

Lemma 2.34. For any $g \in \hat{X}$, the functional $n_{\hat{K}, g}: \hat{X}_{\hat{K}}(g) \rightarrow[0,+\infty)$ is homogeneous in the sense that

for all $z \in \hat{X}_{\hat{K}}(g)$ and $\gamma \in \mathbb{R}$.

$$
n_{\hat{K}, g}(\gamma z)=|\gamma| n_{\hat{K}, g}(z)
$$


Proof. Let us fix some $z \in \hat{X}_{\hat{K}}(g)$ and $\gamma \in \mathbb{R}, \gamma \neq 0$. According to formula (2.40), we have

$$
n_{\hat{K}, g}(\gamma z)=\sqrt{2} \inf \left\{|\lambda| \mid \lambda \in \mathbb{C} \wedge \gamma z \in \hat{X}_{\hat{K}, \lambda}(g)\right\} .
$$

Applying assertion (iii) of Lemma 2.33 with $\alpha=1 / \gamma$, we conclude that an element $\gamma z$ belongs to $\hat{X}_{\hat{K}, \lambda}(g)$ if, and only if $z \in \hat{X}_{\hat{K}, \lambda|\gamma|^{-1}}(g)$. Therefore, equality (2.41) can be rewritten as

$$
\begin{aligned}
n_{\hat{K}, g}(\gamma z) & =\sqrt{2} \inf \left\{|\lambda| \mid \lambda \in \mathbb{C} \wedge \gamma z \in \hat{X}_{\hat{K}, \lambda}(g)\right\} \\
& =\sqrt{2} \inf \left\{|\lambda| \mid \lambda \in \mathbb{C} \wedge z \in \hat{X}_{\hat{K}, \lambda /|\gamma|}(g)\right\} \\
& =|\gamma| \sqrt{2} \inf \left\{\frac{\lambda}{|\gamma|} \mid \lambda \in \mathbb{C} \wedge z \in \hat{X}_{\hat{K}, \lambda /|\gamma|}(g)\right\} \\
& =|\gamma| \sqrt{2} \inf \left\{|\mu| \mid \mu \in \mathbb{C} \wedge z \in \hat{X}_{\hat{K}, \mu}(g)\right\}=|\gamma| n_{\hat{K}, g}(z),
\end{aligned}
$$

as required.

We are interested mainly in elements of $\hat{X}$ that are $\hat{f}$-measurable with respect to $\hat{K}$ for a suitably chosen $f$ from $X$ (actually, from $[K \cup(-K)] \backslash K^{\diamond}$ because otherwise, by Proposition 2.11, there are no $f$-measurable elements outside $K^{\diamond}$ ). In this case, it is convenient to use the following formulae for computation of value (2.40).

Lemma 2.35. Let $f \in X$ and let $\{x, y\} \subset X$ be some elements $f$-measurable with respect to $K$. Then the formulae

$$
n_{\hat{K}, \hat{f}}(x+\mathrm{i} y)=\sqrt{2} \inf \{\varrho \in[0,+\infty) \mid \exists \vartheta \in[-\pi, \pi]:(2.30) \text { and (2.31) hold }\}
$$

and

$$
n_{\hat{K}, \hat{f}}(x+\mathrm{i} y)=\inf \{r \in[0,+\infty) \mid \exists \omega \in[-\pi, \pi]: \text { (2.26) and (2.27) hold }\}
$$

are true.

Proof. By Lemma 2.31, the element $x+\mathrm{i} y$ is $\hat{f}$-measurable with respect to $\hat{K}$ and, therefore, the value of $n_{\hat{K}, g}(x+\mathrm{i} y)$ is finite. In view of formula (2.29) established in the proof of Lemma 2.29, the relation

$$
-\lambda \hat{f} \leqq_{\hat{K}} x+\mathrm{i} y \leqq_{\hat{K}} \lambda \hat{f}
$$

with $\lambda=\varrho \mathrm{e}^{\mathrm{i} \vartheta}$ is equivalent to the system of order inequalities (2.30), (2.31). Therefore, definition (2.40) of the mapping $n_{\hat{K}, \hat{f}}$ yields the required equality (2.42).

Formula (2.43) is a consequence of (2.42). Indeed, as is shown in the proof of Lemma 2.29, there is a one-to-one correspondence between systems (2.30), (2.31) and (2.26), (2.27), with

$$
\frac{r}{\varrho}=\sqrt{2},
$$

and it suffices to use Lemma 2.34. 
The best constant $r$ in relations (2.26) and (2.27) satisfied by the respective components $x$ and $y$ of an $\hat{f}$-measurable element $x+\mathrm{i} y$ is determined by the value of functional (2.40). More precisely, we have

Lemma 2.36. Let $\{f, x, y\} \subset X$ and

$$
n_{\hat{K}, \hat{f}}(x+\mathrm{i} y)=: r<+\infty .
$$

Then there exists an $\omega \in[-\pi, \pi]$ such that relations (2.26) and (2.27) are satisfied.

Proof. The definition of the functional $n_{\hat{K}, \hat{f}}$ and Lemmata 2.29 and 2.35 yield the existence of sequences $\left(r_{k}\right)_{k=1}^{+\infty} \subset[0,+\infty)$ and $\left(\omega_{k}\right)_{k=1}^{+\infty} \subset[-\pi, \pi]$ such that $\lim _{k \rightarrow+\infty} r_{k}=$ $r$ and the relations

$$
\begin{aligned}
& -r_{k} f \sin \omega_{k} \leqq_{K} x \leqq_{K} r_{k} f \sin \omega_{k}, \\
& -r_{k} f \cos \omega_{k} \leqq_{K} y \leqq_{K} r_{k} f \cos \omega_{k}
\end{aligned}
$$

are true for all $k \in \mathbb{N}$. The compact real sequence $\left(\omega_{k}\right)_{k=1}^{+\infty}$ contains a subsequence $\left(\omega_{k_{j}}\right)_{j=1}^{+\infty}$ convergent to a number $\omega \in[-\pi, \pi]$. Putting $k=k_{j}$ in (2.44) and (2.45), passing to the limit as $j \rightarrow+\infty$, and taking into account the fact that $K$ is a closed subset of $X$, we arrive at relations (2.26) and (2.27).

The following statement is an extension of Lemma 2.24 to the complex case.

Lemma 2.37. Let $f \in X$ and $z \in \hat{X}$. Then $n_{\hat{K}, \hat{f}}(z)=0$ if, and only if $z \in K^{\diamond}+\mathrm{i} K^{\diamond}$.

Proof. Let us suppoose that $z=x+\mathrm{i} y$, where $\{x, y\} \subset K^{\diamond}$. According to formula (2.43) of Lemma 2.35, we have

$$
\begin{aligned}
n_{\hat{K}, \hat{f}}(x+\mathrm{i} y) & =\inf \{r \in[0,+\infty) \mid \exists \omega \in[-\pi, \pi]:(2.26) \text { and }(2.27) \text { hold }\} \\
& \leq \inf \left\{r \in[0,+\infty) \mid \exists\{\sigma, \kappa\} \subset\{-1,1\}:-\sigma r f \varliminf_{K} x \sqrt{2} \leqq_{K} \sigma r f\right. \\
& \left.\quad \text { and }-\kappa r f \varliminf_{K} y \sqrt{2} \leqq_{K} \kappa r f\right\} \\
& \leq \inf \left\{r \in[0,+\infty) \mid-\sigma r f \leqq_{K} x \sqrt{2} \leqq_{K} \sigma r f \text { with some } \sigma \in\{-1,1\}\right\} \\
& =\inf \left\{|\alpha| \mid \alpha \in \mathbb{R} \wedge-\alpha f \leqq_{K} x \sqrt{2} \leqq_{K} \alpha f\right\} \\
& =n_{K, f}(x \sqrt{2}) .
\end{aligned}
$$

By virtue of Lemma 2.34, we have

$$
n_{K, f}(x \sqrt{2})=\sqrt{2} n_{K, f}(x) .
$$

However, in view of Lemma 2.24, $n_{K, f}(x)=0$ and, therefore, by (2.46), the nonnegative number $n_{\hat{K}, \hat{f}}(x+\mathrm{i} y)$ is equal to 0 .

Assume now that $n_{\hat{K}, \hat{f}}(x+\mathrm{i} y)=0$. By virtue of Lemma 2.36, there exists an $\omega \in$ $[-\pi, \pi]$ such that relations (2.26) and (2.27) are satisfied with $r=0$, i. e., $0 \leqq K \quad x \leqq_{K} 0$ and $0 \leqq_{K} y \leqq_{K} 0$. This means that $\{x, y\} \subset K^{\diamond}$. 
Formula (2.40) allows one to construct a natural extension $n_{\hat{K}, \hat{f}}: \hat{X} \rightarrow[0,+\infty]$ of the mapping $X \ni x \mapsto n_{K, f}(x)$ given by relation (2.13). More precisely, the following statement is true.

Proposition 2.38. Let $f \in X$. Then the equality

$$
n_{\hat{K}, \hat{f}}(x)=n_{K, f}(x)
$$

is true for all $x \in X$.

Proof. First of all, we note that it suffices to consider the case where $f \square_{K} 0$ because in the contrary case, by Proposition 2.11, we have $X_{K}(f)=K^{\diamond}$ and, therefore, in view of Lemmata 2.24 and 2.37, both $n_{K, f}$ and $n_{\hat{K}, \hat{f}}$ vanish on the set $X_{K}(f)$.

For the sake of definiteness, we assume that $f \geqq_{K} 0$. Setting $y=0$ in formula (2.43) of Lemma 2.35, we obtain

$$
\begin{aligned}
n_{\hat{K}, \hat{f}}(x) & =\inf \{r \in[0,+\infty) \mid \exists \omega \in[-\pi, \pi]: \cos \omega \geq 0 \text { and (2.26) holds }\} \\
& =\inf \left\{r \in[0,+\infty) \mid \exists \omega \in\left[-\frac{\pi}{2}, \frac{\pi}{2}\right]:(2.26) \text { holds }\right\} .
\end{aligned}
$$

Since the mapping $\sin :\left[-\frac{\pi}{2}, \frac{\pi}{2}\right] \rightarrow[-1,1]$ is a bijection, we see that $(2.48)$ can be rewritten in the form

$$
\begin{aligned}
n_{\hat{K}, \hat{f}}(x) & =\inf \left\{r \in[0,+\infty) \mid \exists h \in[-1,1]:-r h f \leqq_{K} x \leqq_{K} r h f\right\} \\
& =\inf \left\{|\alpha| \in[0,+\infty) \mid-\alpha f \leqq_{K} x \leqq_{K} \alpha f\right\},
\end{aligned}
$$

which, by virtue of (2.13), proves that equality (2.47) is true for all $x$ from $X_{K}(f)$. In the case where $x$ is not $f$-measurable with respect to $K$, by Lemma 2.31, both values are equal to $+\infty$.

Proposition 2.38 is, in fact, a particular case of a more general result. Namely, the following statement is true.

Proposition 2.39. Let $f \in X$. For arbitrary $\{x, y\} \subset X$ which are $f$-measurable with respect to the wedge $K$, the equality

$$
n_{\hat{K}, \hat{f}}(x+\mathrm{i} y)=\sqrt{\left(n_{K, f}(x)\right)^{2}+\left(n_{K, f}(y)\right)^{2}}
$$

is true.

Formula (2.49) resembles to some extent the Pythagorean formula, with $n_{K, f}(x)$, $n_{K, f}(y)$, and $n_{\hat{K}, \hat{f}}(x+\mathrm{i} y)$, respectively, playing the roles of catheti and hypotenuse. The proof of Proposition 2.39 is omitted.

2.5.4. Measuring rotated elements. In the sequel, we need to compute the values of the functional $n_{\hat{K}, \hat{f}}$ on elements of the form $\mathrm{e}^{\mathrm{i} t}(x+\mathrm{i} y)$, where $t \in[-\pi, \pi]$ is arbitrary and $\{x, y\} \subset X_{K}(f)$ with some $f$ satisfying the condition $f \square_{K} 0$. 
Definition 2.40. Given an $f \in X$, we put

$$
R_{\hat{K}, \hat{f}}(x+\mathrm{i} y):=\inf _{t \in[-\pi, \pi]} n_{\hat{K}, \hat{f}}\left(\mathrm{e}^{\mathrm{i} t}(x+\mathrm{i} y)\right)
$$

if $\{x, y\} \subset X$ are $f$-measurable with respect to $K$, and set formally $R_{\hat{K}, \hat{f}}(x+\mathrm{i} y):=+\infty$ in the contrary case.

It follows from Lemma 2.32 that the right-hand side of (2.50) is finite for arbitrary $\{x, y\} \subset X_{K}(f)$ and $t \in[-\pi, \pi]$ and, thus, Definition 2.40 makes sense.

Lemma 2.41. Let $f \in X$ and $\{x, y\} \subset X_{K}(f)$. Then, for an arbitrary $\varphi \in[-\pi, \pi]$, the equality

is true.

$$
R_{\hat{K}, \hat{f}}\left(\mathrm{e}^{\mathrm{i} \varphi}(x+\mathrm{i} y)\right)=R_{\hat{K}, \hat{f}}(x+\mathrm{i} y)
$$

Proof. According to formula (2.50), we have

$$
\begin{aligned}
R_{\hat{K}, \hat{f}}\left(\mathrm{e}^{\mathrm{i} \varphi}(x+\mathrm{i} y)\right) & =\inf _{t \in[-\pi, \pi]} n_{\hat{K}, \hat{f}}\left(\mathrm{e}^{\mathrm{i} \varphi} \mathrm{e}^{\mathrm{i} t}(x+\mathrm{i} y)\right) \\
& =\inf _{t \in[-\pi, \pi]} n_{\hat{K}, \hat{f}}\left(\mathrm{e}^{\mathrm{i}(t+\varphi)}(x+\mathrm{i} y)\right) .
\end{aligned}
$$

Let us take an arbitrary $\varphi \in\left[-\frac{\pi}{2}, \frac{\pi}{2}\right]$ and put

$$
\varphi_{t}:= \begin{cases}t+\varphi+\pi & \text { if } t+\varphi<-\pi, \\ t+\varphi & \text { if }-\pi \leq t+\varphi \leq \pi, \\ t+\varphi-\pi & \text { if } t+\varphi>\pi\end{cases}
$$

for all $t$ from $[-\pi, \pi]$. It is clear that $\left\{\mathrm{e}^{\mathrm{i} \varphi_{t}} \mid t \in[-\pi, \pi]\right\}=\{\lambda \in \mathbb{C}|| \lambda \mid=1\}$ for any $\varphi$. Therefore, equality (2.52) yields

$$
\begin{aligned}
R_{\hat{K}, \hat{f}}\left(\mathrm{e}^{\mathrm{i} \varphi}(x+\mathrm{i} y)\right) & =\inf _{t \in[-\pi, \pi]} n_{\hat{K}, \hat{f}}\left(\mathrm{e}^{\mathrm{i} \varphi t}(x+\mathrm{i} y)\right) \\
& =\inf _{t \in[-\pi, \pi]} n_{\hat{K}, \hat{f}}\left(\mathrm{e}^{\mathrm{i} t}(x+\mathrm{i} y)\right)=R_{\hat{K}, \hat{f}}(x+\mathrm{i} y) .
\end{aligned}
$$

Applying formula (2.51) sequentially, we prove that it is true with arbitrary values of $\varphi$ from $[-\pi, \pi]$.

Together with Lemma 2.41, the next statement is a basic tool in the proof of Theorem 3.1 from Section 3.

Lemma 2.42. Let $f \in X,\{x, y\} \subset X_{K}(f)$, and

$$
R:=R_{\hat{K}, \hat{f}}(x+\mathrm{i} y) .
$$

Then there exist some $\left\{\vartheta_{*}, \omega_{*}\right\} \subset[-\pi, \pi]$ such that the relations

$$
\begin{aligned}
& -R f \sin \omega_{*} \leqq_{K} x \cos \vartheta_{*}-y \sin \vartheta_{*} \leqq_{K} R f \sin \omega_{*}, \\
& -R f \cos \omega_{*} \leqq_{K} x \sin \vartheta_{*}+y \cos \vartheta_{*} \leqq_{K} R f \cos \omega_{*}
\end{aligned}
$$


hold. Moreover, if $R>0$, then there do not exist any numbers $\varepsilon \in(0, R)$ and $\{\tilde{\vartheta}, \tilde{\omega}\} \subset$ $[-\pi, \pi]$ for which the inequalities

$$
\begin{aligned}
& -(R-\varepsilon) f \sin \tilde{\omega} \leqq_{K} x \cos \tilde{\vartheta}-y \sin \tilde{\vartheta} \leqq_{K}(R-\varepsilon) f \sin \tilde{\omega}, \\
& -(R-\varepsilon) f \cos \tilde{\omega} \leqq_{K} x \sin \tilde{\vartheta}+y \cos \tilde{\vartheta} \leqq_{K}(R-\varepsilon) f \cos \tilde{\omega}
\end{aligned}
$$

would be satisfied.

Proof. Let us fix some $\{x, y\} \subset X_{K}(f)$ and define $R$ by (2.53). Then

$$
R=\inf _{t \in[-\pi, \pi]} r_{t},
$$

where $r_{t}:=n_{\hat{K}, \hat{f}}\left(\mathrm{e}^{\mathrm{i} t}(x+\mathrm{i} y)\right)$ for all $t \in[-\pi, \pi]$. By virtue of Lemma 2.32, we have $0 \leq R<+\infty$.

Taking Lemma 2.36 and formula (2.38) into account, we conclude that, with any $t \in[-\pi, \pi]$, one can associate an $\omega_{t} \in[-\pi, \pi]$ for which

$$
-r_{t} f \sin \omega_{t} \leqq_{K} x \cos t-y \sin t \leqq_{K} r_{t} f \sin \omega_{t}
$$

and

$$
-r_{t} f \cos \omega_{t} \leqq_{K} y \cos t+x \sin t \leqq_{K} r_{t} f \cos \omega_{t} .
$$

By virtue of (2.58), there exists a sequence $\left(t_{m}\right)_{m=1}^{+\infty} \subset[-\pi, \pi]$ such that

$$
\lim _{m \rightarrow+\infty} r_{t_{m}}=R \text {. }
$$

Being bounded, this sequence contains a subsequence convergent to a certain $\vartheta_{*} \in$ $[-\pi, \pi]$. We can assume, without loss of generality, that such a subsequence has already been selected and, thus, in addition to (2.61), we have

$$
\lim _{m \rightarrow+\infty} t_{m}=\vartheta_{*}
$$

On the other hand, the sequence $\left(\omega_{t_{m}}\right)_{m=1}^{+\infty} \subset[-\pi, \pi]$ is also bounded and, therefore, there exists a sequence $\left(m_{j}\right)_{j=1}^{+\infty} \subset \mathbb{N}$ such that $\lim _{j \rightarrow+\infty} \omega_{t_{m_{j}}}=\omega_{*}$ with a certain $\omega_{*} \in[-\pi, \pi]$. Setting $t=t_{m_{j}}$ in (2.59) and (2.60), passing to the limit as $j$ tends to $+\infty$, and using (2.61), (2.62), and the fact that $K$ is a closed set, we arrive at relations (2.54) and (2.55) with the above values of $\vartheta_{*}$ and $\omega_{*}$.

Assume now that $R>0$ and relations (2.56) and (2.57) are satisfied with some $\varepsilon$, $0 \leq \varepsilon<R$, and $\{\tilde{\vartheta}, \tilde{\omega}\} \subset[-\pi, \pi]$. Due to formula (2.43) of Lemma 2.35 and equality (2.38) from the proof of Lemma 2.32, relations (2.56) and (2.57) imply that $r_{t} \leq R-\varepsilon$ for all $t \in[-\pi, \pi]$, whence the estimate

$$
\inf _{t \in[-\pi, \pi]} r_{t} \leq R-\varepsilon
$$

follows. However, by virtue of (2.58), inequality (2.63) yields $R \leq R-\varepsilon$ and, therefore, $\varepsilon=0$.

The property described by Lemma 2.37 is also true for functional (2.50). 
Lemma 2.43. Let $f \in X$ and $z \in \hat{X}$. Then $R_{\hat{K}, \hat{f}}(z)=0$ if, and only if $z \in K^{\diamond}+\mathrm{i} K^{\diamond}$.

Proof. The inclusion $z \in K^{\diamond}+\mathrm{i} K^{\diamond}$ means that the element $z$ is 0 -measurable with respect to $\hat{K}$. In this case, Lemma 2.32 guarantees that so does the element $\mathrm{e}^{\mathrm{i} t} z$ with any $t$ from $[-\pi, \pi]$ and, hence, by Lemma 2.37 , it follows that $n_{\hat{K}, \hat{f}}\left(\mathrm{e}^{\mathrm{i} t} z\right)=0$ for all $t \in[-\pi, \pi]$. Relation (2.50) then yields $R_{\hat{K}, \hat{f}}(z)=0$.

Conversely, if $R_{\hat{K}, \hat{f}}(x+\mathrm{i} y)=0$, then, by Lemma 2.42 , there exists some $\vartheta$ from $[-\pi, \pi]$ such that

$$
\begin{aligned}
& 0 \leqq_{K} x \cos \vartheta-y \sin \vartheta \leqq_{K} 0, \\
& 0 \leqq_{K} x \sin \vartheta+y \cos \vartheta \leqq_{K} 0 .
\end{aligned}
$$

According to formula (2.38), this means that the element $\mathrm{e}^{\mathrm{i} \vartheta}(x+\mathrm{i} y)$ is 0 -measurable with respect to $\hat{K}$ and, thus, by Lemma 2.32 , so does the element $x+\mathrm{i} y$.

2.6. Linear operators vanishing on the blade of a wedge. For the sake of brevity, we introduce the following definition [11].

Definition 2.44. We say that an eigenvalue $\lambda$ of a bounded linear operator $A$ : $X \rightarrow X$ is substantial with respect to the wedge $K$ (or, shortly, $K$-substantial) if $\lambda$ is non-zero and at least one eigenvector not belonging to $K^{\diamond}+\mathrm{i} K^{\diamond}$ corresponds to it.

As usual (see, e. g., [5]), by a complex eigenvalue $\lambda \in \mathbb{C}$ of a bounded linear operator $A: X \rightarrow X$ acting in a real Banach space $X$, the eigenvalue of its complexification $\hat{A}=A+\mathrm{i} A: \hat{X} \rightarrow \hat{X}$ is meant, where

$$
\hat{A}(x+\mathrm{i} y):=A x+\mathrm{i} A y
$$

for all $\{x, y\} \subset X$.

Example 2.45. All the eigenvalues of a bounded linear operator $A: X \rightarrow X$ are substantial with respect to an arbitrary cone in $X$.

We devote our present study mostly to the linear operators $A: X \rightarrow X$ vanishing on the blade of a proper wedge $K$, i. e., such that

$$
K^{\diamond} \subseteq \operatorname{ker} A \text {. }
$$

Example 2.46. If $K$ is a cone, then condition (2.65) is satisfied in an obvious way for every linear operator $A: X \rightarrow X$.

In the general case, the restrictiveness of condition (2.65) imposed on the operator $A$ grows with the "width" of $K^{\diamond}$.

Example 2.47. Let us consider the set

$$
C_{\Omega, \sigma}([a, b], \mathbb{R})=\{x \in C([a, b], \mathbb{R}) \mid \sigma x([a, b] \backslash \Omega) \subseteq[0,+\infty)\},
$$

where $\Omega$ is a certain subset of $[a, b]$ such that $[a, b] \backslash \Omega$ is closed, and $\sigma \in\{-1,1\}$. The set $C_{\Omega, \sigma}([a, b], \mathbb{R})$ is obviously a closed wedge in the Banach space $C([a, b], \mathbb{R})$ of all the continuous scalar functions on the bounded interval $[a, b]$. This wedge is 
solid because, as one can show, its interior is constituted by the continuous functions $x:[a, b] \rightarrow \mathbb{R}$ such that $\sigma x([a, b] \backslash \Omega) \subseteq(0,+\infty)$.

Consider the operator $A: C([a, b], \mathbb{R}) \rightarrow C([a, b], \mathbb{R})$ given by the formula

$$
(A x)(t)=\int_{\tau}^{t} k(t, s) x(\omega(s)) d s, \quad t \in[a, b],
$$

in which $\omega:[a, b] \rightarrow[a, b]$ is a measurable function, whereas the function $k:$ $[a, b] \times[a, b] \rightarrow \mathbb{R}$ is continuous in the first variable and Lebesgue integrable in the second one. The operator $A$ vanishes on the blade of the wedge $C_{\Omega, \sigma}([a, b], \mathbb{R})$ when $\omega$ satisfies the condition

$$
\omega([a, b]) \subseteq[a, b] \backslash \Omega .
$$

Indeed, the blade of $C_{\Omega, \sigma}([a, b], \mathbb{R})$ consists of those continuous functions $x$ : $[a, b] \rightarrow \mathbb{R}$ such that

$$
x(t)=0 \quad \text { for all } t \in[a, b] \backslash \Omega .
$$

If $\omega$ is such that condition (2.67) holds, then $A x$ is equal identically to zero for every function $x$ satisfying condition (2.68), i. e., the relation $x \in\left(C_{\Omega, \sigma}([a, b], \mathbb{R})\right)^{\diamond}$ implies that $A x=0$. This means that (2.65) is true for $K=C_{\Omega, \sigma}([a, b], \mathbb{R})$ and $A$ given by (2.66).

Our interest to the property described by condition (2.65) is motivated by the following statement.

Lemma 2.48. Assume that $A: X \rightarrow X$ is a linear operator vanishing on the blade of a wedge $K \subseteq X$. Then every non-zero eigenvalue of $A$ is $K$-substantial.

Proof. Let $\lambda$ be an arbitrary non-zero eigenvalue of $A$. Then there exists some non-zero element $w$ from $\hat{X}$ such that

$$
\lambda w=\hat{A} w .
$$

Assume that, on the contrary, $\lambda$ is not $K$-substantial and, therefore, according to Definition 2.44, every eigenvector $w$ in (2.69) belongs to $K^{\diamond}+\mathrm{i} K^{\diamond}$. By virtue of inclusion (2.65), this yields $\hat{A} w=0$, and, hence, by (2.69), $w=0$, which is impossible because $w$ is an eigenvector of $\hat{A}$. The contradiction obtained proves our lemma.

Assumption (2.65) may seem to be unnecessarily strong because, in fact, it guarantees that not only some eigenvectors corresponding to non-zero eigenvalues of $A$ do not belong to the blade of $\hat{K}$ but all such eigenvectors possess this property. Note however that, in the theorems of Sections 3 and 4, condition (2.65) cannot be dropped even in the two-dimensional case (see Example 5.1).

\section{UPPER BOUNDS FOR $K$-SUBSTANTIAL EIGENVALUES}

The following theorem provides an upper bound for $K$-substantial eigenvalues of a sufficiently wide class of linear operators in a real Banach space $X$. 
Theorem 3.1. Let $K$ be a proper wedge in $X$ and $A_{1}: X \rightarrow X, A_{2}: X \rightarrow X$ be bounded linear operators such that

$$
A_{1}(K) \cup A_{2}(K) \subseteq K .
$$

Assume also that the relation

$$
A_{1} f+A_{2} f \leqq_{K} \alpha f
$$

is true with some $\alpha \in[0,+\infty)$ and $f \in X$ for which

$$
f \varepsilon_{K ; H} 0,
$$

where $H$ is a certain linear manifold in $X$ satisfying the inclusion

$$
H \supseteq \operatorname{im}\left(A_{1}-A_{2}\right) \text {. }
$$

Then every $K$-substantial eigenvalue $\lambda$ of the operator $A_{1}-A_{2}$ admits the estimate

$$
|\lambda| \leq \alpha .
$$

In (3.1), (3.4), and similar relations, we use the standard notation

$$
A(M):=\{A x \mid x \in M\},
$$

where $M \subseteq X$. Recall that the binary relation " $\varepsilon_{K ; H}$ " appearing in (3.3) is introduced on $X$ by Definition 2.16 .

Proof of Theorem 3.1. Let $\lambda=\varrho \mathrm{e}^{\mathrm{i} \vartheta}, \varrho \in(0,+\infty)$, be a $K$-substantial eigenvalue of the complexification $\hat{A}=\hat{A}_{1}-\hat{A}_{2}$ of the operator

$$
A:=A_{1}-A_{2} \text {. }
$$

In view of Definition 2.44 and equality (2.23), there exists an element $w=x+\mathrm{i} y$ such that $\{x, y\} \subset X,\{x, y\} \not \subset K^{\diamond}$, and equality (2.69) holds.

We divide the present proof into several parts.

Claim 1. The element $w$ is $\hat{f}$-measurable with respect to $\hat{K}$.

Indeed, equality (2.69) means that

$$
\varrho w=\mathrm{e}^{-\mathrm{i} \vartheta} \hat{A} w .
$$

According to formulae (2.38) and (2.64), we have

$$
\mathrm{e}^{-\mathrm{i} \vartheta} \hat{A} w=A x \cos \vartheta+A y \sin \vartheta+\mathrm{i}(A y \cos \vartheta-A x \sin \vartheta)
$$

and, therefore, (3.7) can be rewritten as the system

$$
\begin{aligned}
& \varrho x=A x \cos \vartheta+A y \sin \vartheta, \\
& \varrho y=A y \cos \vartheta-A x \sin \vartheta
\end{aligned}
$$

By virtue of assumption (3.4), it follows from (3.8) and (3.9) that $x$ and $y$ both lie in $H$ (to prove this, it suffices to use the linearity of the set $H$ ). However, according to Definition 2.12, condition (3.3) means that all the elements from $H$ are $f$-measurable 
with respect to $K$ and, hence, by Lemma 2.31, the element $x+\mathrm{i} y$ is $(f+\mathrm{i} f)$-measurable with respect to $\hat{K}$.

Claim 2. The number

is strictly positive.

$$
R:=R_{\hat{K}, \hat{f}}(x+\mathrm{i} y)
$$

Indeed, by Claim 1 and Lemma 2.41, the right-hand side of (3.10) is a finite number. Since $\{x, y\} \not \subset K^{\diamond}$, Lemma 2.37 yields $R>0$.

Claim 3. The elements $x$ and $y$ satisfy the equalities

$$
\begin{aligned}
& A x=\varrho(x \cos \vartheta-y \sin \vartheta), \\
& A y=\varrho(x \sin \vartheta+y \cos \vartheta) .
\end{aligned}
$$

According to formula (2.38), system (3.11), (3.12) is an equivalent form of relation (2.69) satisfied by $w$.

Claim 4. There exist some $\omega_{*}$ and $t_{*}$ from $[-\pi, \pi]$ such that (2.54) and (2.55) are true for $x$ and $y$, and there do not exist any $\{\tilde{\omega}, \tilde{\vartheta}\} \subset[-\pi, \pi]$ for which the relations

$$
\begin{aligned}
& -r f \sin \tilde{\omega} \leqq_{K} x \cos \tilde{\vartheta}-y \sin \tilde{\vartheta} \leqq_{K} r f \sin \tilde{\omega}, \\
& -r f \cos \tilde{\omega} \leqq_{K} x \sin \tilde{\vartheta}+y \cos \tilde{\vartheta} \leqq_{K} r f \cos \tilde{\omega}
\end{aligned}
$$

would be satisfied with some $r \in(0, R)$.

This statement is an immediate consequence of formula (3.10) and Lemma 2.42.

CLAIM 5. There is an $\Omega$ from $[-\pi, \pi]$ such that the relations

$$
\begin{aligned}
& -\alpha R f \sin \Omega \leqq_{K} A x \cos \vartheta_{*}-A y \sin \vartheta_{*} \leqq_{K} \alpha R f \sin \Omega, \\
& -\alpha R f \cos \Omega \leqq_{K} A x \sin \vartheta_{*}+A y \cos \vartheta_{*} \leqq_{K} \alpha R f \cos \Omega,
\end{aligned}
$$

are true, where $A$ is given by (3.6).

In view of assumption (3.1), both operators $A_{1}$ and $A_{2}$ preserve order inequalities. Therefore, relations (2.54) and (2.55), together with Lemma 2.6, yield

$$
\begin{aligned}
& -R A_{j} f \sin \omega_{*} \leqq_{K} \sigma\left[A_{j} x \cos \vartheta_{*}-A_{j} y \sin \vartheta_{*}\right] \leqq_{K} R A_{j} f \sin \omega_{*}, \\
& -R A_{j} f \cos \omega_{*} \leqq_{K} x\left[A_{j} x \sin \vartheta_{*}+A_{j} y \cos \vartheta_{*}\right] \leqq_{K} R A_{j} f \cos \omega_{*}
\end{aligned}
$$

for all $j=1,2$ and $\{\sigma, \varkappa\} \subset\{-1,1\}$. Summing the two relations obtained from (3.17) with $j=1, \sigma=1$ and $j=2, \sigma=-1$, respectively, we obtain

$-R\left(A_{1}+A_{2}\right) f \sin \omega_{*} \leqq_{K}\left(A_{1}-A_{2}\right) x \cos \vartheta_{*}-\left(A_{1}-A_{2}\right) y \sin \vartheta_{*} \leqq_{K} R\left(A_{1}+A_{2}\right) f \sin \omega_{*}$, i. e.,

$$
-R\left(A_{1}+A_{2}\right) f \sin \omega_{*} \leqq_{K} A x \cos \vartheta_{*}-A y \sin \vartheta_{*} \leqq_{K} R\left(A_{1}+A_{2}\right) f \sin \omega_{*} .
$$

In a similar manner, putting in (3.18) $j=1, \varkappa=1$ and $j=2, \varkappa=-1$ and summing the resulting two relations, we get

$$
-R\left(A_{1}+A_{2}\right) f \cos \omega_{*} \leqq_{K} A x \sin \vartheta_{*}+A y \cos \vartheta_{*} \leqq_{K} R\left(A_{1}+A_{2}\right) f \cos \omega_{*} .
$$


Let us consider the following four cases.

Case 1. $\sin \omega_{*} \geq 0$ and $\cos \omega_{*} \geq 0$.

Using assumption (3.2) in relations (3.19), (3.20) and putting $\Omega:=\omega_{*}$, we arrive immediately at (3.15), (3.16).

Case 2. $\sin \omega_{*} \geq 0$ and $\cos \omega_{*}<0$.

Recall that, by assumption, $f \geqq_{K} 0$ and, due to condition (3.1), $A_{1} f+A_{2} f \geqq_{K} 0$. In view of assertion (iii) of Lemma 2.7, relation (3.20) and Claim 2 imply that, in this case,

$$
0 \leqq_{K} A x \sin \vartheta_{*}+A y \cos \vartheta_{*} \leqq_{K} 0 .
$$

whereas the term $\left(A_{1}+A_{2}\right) f \sin \omega_{*}$, by virtue of (3.2), admits the estimate

$$
\left(A_{1}+A_{2}\right) f \sin \omega_{*} \leqq_{K} \alpha f .
$$

Therefore, (3.15) and (3.16) are satisfied with $\Omega:=\frac{\pi}{2}$.

Case 3. $\sin \omega_{*}<0$ and $\cos \omega_{*} \geq 0$.

Relation (3.19) now yields

$$
0 \leqq_{K} A x \cos \vartheta_{*}-A y \sin \vartheta_{*} \leqq_{K} 0
$$

and, similarly to Case 2, we conclude that (3.15) and (3.16) hold with $\Omega:=0$.

Case 4. $\sin \omega_{*}<0$ and $\cos \omega_{*}<0$.

A reasoning analogous to those presented above show that, in this case, system (3.19), (3.20) has form (3.21), (3.22) and, therefore, relations (3.15) and (3.16) are satisfied both with $\Omega=\frac{\pi}{2}$ and $\Omega=0$. This proves our Claim 5 .

Having established the facts above, we now turn to the proof of estimate (3.5).

According to Claim 3, the components $x$ and $y$ of the eigenvector $w$ of $\hat{A}$ satisfy equalities (3.11) and (3.12). Therefore,

$$
\begin{aligned}
A x \cos \vartheta_{*}-A y \sin \vartheta_{*}= & \varrho(x \cos \vartheta-y \sin \vartheta) \cos \vartheta_{*}-\varrho(x \sin \vartheta+y \cos \vartheta) \sin \vartheta_{*} \\
= & \varrho\left[\cos \vartheta \sin \vartheta_{*}-\sin \vartheta \sin \vartheta_{*}\right] x \\
& -\varrho\left[\sin \vartheta \cos \vartheta_{*}+\cos \vartheta \sin \vartheta_{*}\right] y \\
= & \varrho x \cos \left(\vartheta+\vartheta_{*}\right)-\varrho y \sin \left(\vartheta+\vartheta_{*}\right)
\end{aligned}
$$

and, similarly,

$$
\begin{aligned}
A x \sin \vartheta_{*}+A y \cos \vartheta_{*}= & \varrho(x \cos \vartheta-y \sin \vartheta) \sin \vartheta_{*}+\varrho(x \sin \vartheta+y \cos \vartheta) \cos \vartheta_{*} \\
= & \varrho\left[\cos \vartheta \sin \vartheta_{*}+\sin \vartheta \cos \vartheta_{*}\right] x \\
& +\varrho\left[\cos \vartheta \cos \vartheta_{*}-\sin \vartheta \sin \vartheta_{*}\right] y \\
= & \varrho x \sin \left(\vartheta+\vartheta_{*}\right)+\varrho y \cos \left(\vartheta+\vartheta_{*}\right)
\end{aligned}
$$


Applying these formulae to the corresponding expressions in (3.15) and (3.16) and taking the inequality $\varrho>0$ into account, we obtain

$$
\begin{gathered}
-\frac{\alpha R}{\varrho} f \sin \Omega \leqq_{K} x \cos \left(\vartheta+\vartheta_{*}\right)-y \sin \left(\vartheta+\vartheta_{*}\right) \leqq_{K} \frac{\alpha R}{\varrho} f \sin \Omega, \\
-\frac{\alpha R}{\varrho} f \cos \Omega \leqq_{K} x \sin \left(\vartheta+\vartheta_{*}\right)+y \cos \left(\vartheta+\vartheta_{*}\right) \leqq_{K} \frac{\alpha R}{\varrho} f \cos \Omega .
\end{gathered}
$$

System (3.23), (3.24), obviously, has form (3.13), (3.14) with $\tilde{\vartheta}:=\vartheta+\vartheta_{*}, \tilde{\omega}:=\Omega$, and $r:=\alpha R / \varrho$. In view of Claim 4 , it now follows that

$$
\frac{\alpha R}{\varrho} \geq R
$$

whence, by Claim 2, we arrive at the inequality $\varrho \leq \alpha$. Recalling that $\varrho=|\lambda|$, we conclude that the required estimate (3.5) holds.

The assumption that $f$ should lie in $K$ and be different from zero in Theorem 3.1 is motivated by Proposition 2.22.

Remark 3.2. A linear operator $A: X \rightarrow X$ admits representation in form (1.4), where $A_{1}$ and $A_{2}$ are linear mappings preserving $K$, if and only if there exists a linear operator $B: X \rightarrow X$ such that

$$
B(K) \subseteq K
$$

and

$$
A x \leqq_{K} B x \quad \text { for all } x \in K .
$$

Indeed, (1.4) implies that

$$
A x \leqq_{K} A_{1} x \varliminf_{K} A_{1} x+A_{2} x
$$

for all $x$ such that $x \geqq_{K} 0$ and, therefore, one can set $B:=A_{1}+A_{2}$. Conversely, it follows from (3.25) and (3.26) that the operator $A_{2}:=B-A$ preserves the wedge $K$ and, thus, it remains to put $A_{1}:=B$ in (1.4).

It should be noted that, in the case where the space $X$ is infinite-dimensional, one cannot claim that every bounded linear operator $A: X \rightarrow X$ admits representation (1.4) with bounded linear mappings $A_{1}: X \rightarrow X$ and $A_{2}: X \rightarrow X$ preserving $K$. In particular, in the case where $K$ is a cone which does not possess the property of normality, the classical Theorem 2 of [8] ensures the existence of a continuous (even finite-dimensional) linear operator $A: X \rightarrow X$ that cannot be represented in form (1.4) with bounded $A_{k}: X \rightarrow X, k=1,2$, satisfying condition (3.1).

\section{A theOREM ON THE SPECTRAL RAdiUS OF a COMPaCt OPERATOR}

Theorem 3.1 of Section 3 can be applied to prove the following statement which seems to be useful in studies of the solvability of various linear equations with compact operators. 
Theorem 4.1. Let $X$ be a Banach space over the field $\mathbb{R}, K$ be a proper wedge in $X$, and $A_{1}: X \rightarrow X, A_{2}: X \rightarrow X$ be completely continuous linear operators leaving the wedge $K$ invariant and satisfying the condition

$$
K^{\diamond} \subseteq \operatorname{ker}\left(A_{1}-A_{2}\right) .
$$

In addition, assume that relation (3.2) is satisfied with some constant $\alpha \in[0,+\infty)$ and element $f \in X$ possessing the property

$$
f \varepsilon_{K ; \operatorname{im}\left(A_{1}-A_{2}\right)} 0 .
$$

Then the spectral radius of the operator $A_{1}-A_{2}$ admits the estimate

$$
r\left(A_{1}-A_{2}\right) \leq \alpha
$$

Proof. It follows from the Riesz-Schauder theory (see, e. g., [2]) that, due to the complete continuity of the operator $A_{1}-A_{2}$, its spectrum consists of countably many eigenvalues.

It is easy to see that condition (4.2) implies the existence of a linear manifold $H \subseteq X$ satisfying inclusion (3.4) and such that relation (3.3) holds for the element $f$ (one can put $H:=\operatorname{im}\left(A_{1}-A_{2}\right)$ ). Assumption (4.1), by virtue of Lemma 2.48, implies that every non-zero eigenvalue of the operator $A_{1}-A_{2}$ is $K$-substantial in the sense of Definition 2.44. Therefore, under the conditions assumed, Theorem 3.1 can be used.

Application of Theorem 3.1 guarantees that an arbitrary non-zero eigenvalue $\lambda$ of the operator $A_{1}-A_{2}$ admits estimate (3.5). Considering the least upper bound of $|\lambda|$ in the left-hand side of relation (3.5) with respect to all the non-zero eigenvalues $\lambda$ of $A_{1}-A_{2}$, we arrive immediately at inequality (4.3).

Recall that the binary relation " $\varepsilon_{K ; H}$ " appearing in condition (4.2) has been introduced by Definition 2.16 .

Remark 4.2. The complete continuity of $A_{1}-A_{2}$ in the proof of Theorem 4.1 is used only to guarantee that this operator has discrete spectrum (in fact, an upper bound for the discrete spectrum is established under the conditions specified). Note also that, in the case where $K$ is a solid and normal cone, the assertion of Theorem 4.3 can also be proved by using Theorems 5.3 and 5.5 of [7].

Corollary 4.3. Let $K \subset X$ be a wedge and $A_{1}: X \rightarrow X, A_{2}: X \rightarrow X$ be completely continuous linear operators leaving the wedge $K$ invariant, satisfying condition (4.1) and such that relation (3.2) is true with some $\alpha \in[0,+\infty)$ and element $f \in X$ possessing the property

$$
f \varepsilon_{K} 0 .
$$

Then the spectral radius of the operator $A_{1}-A_{2}$ admits estimate (4.3).

Proof. In view of Lemma 2.13, assumption (4.4) ensures that $f$ possesses property (4.2). Therefore, in order to obtain the required statement, it is sufficient to apply Theorem 4.1. 
For the sake of completeness we note that, by Lemma 2.19, assumption (4.4) is satisfied, in particular, in the classical case where $K$ is solid and the element $f$ belongs its interior.

Remark 4.4. Condition (4.1) for the operator $A: X \rightarrow X$ in Theorem 4.1 and Corollary 4.3 is satisfied automatically if $K$ is a cone.

Corollary 4.5. If $A: X \rightarrow X$ is a completely continuous linear operator leaving invariant a wedge $K$ and, moreover, satisfying condition (2.65) and the relation

$$
\text { Af } \varliminf_{K} \alpha f
$$

with some constant $\alpha \in[0,+\infty)$ and element $f \in X$ possessing property (4.4), then the estimate

$$
r(A) \leq \alpha
$$

is true.

Proof. Corollary 4.5 is an immediate consequence of Corollary 4.3 in the case where $A_{1}=A$ and $A_{2}=0$.

Remark 4.6. A number of theorems that, for a positive operator $A$ preserving a cone $K \subset X$, allow one to deduce estimate (4.6) from relation (4.5) are well-known, e. g., from $[6,7,15]$. Apart from those relying on the $f$-positivity of the operator, in the results mentioned, the element $f$ appearing in (4.5) is supposed either to lie in the interior of $K$ (Theorem 16.2 (b) of [6]) or to be its quasi-interior element (Theorem 16.2 (a) from [6]). It is important to note that condition (4.2), which also expresses a certain kind of the strong positivity* of the element $f$ and, under these circumstances, replaces the two conditions mentioned, depends on the image space of the operator $A_{1}-A_{2}$.

Corollary 4.3 also implies an analogue of Corollary 4.5 for the "negative" operators.

Corollary 4.7. Let $A: X \rightarrow X$ be a completely continuous linear operator such that $A(-K) \subseteq K$ and, moreover, the relation

$$
A f \geqq_{K}-\alpha f
$$

be satisfied with some $\alpha \in[0,+\infty)$ and $f \in X$ possessing property (4.4). Then the spectral radius of $A$ admits estimate (4.6).

Proof. It suffices to put $A_{1}=0$ and $A_{2}=-A$ in Corollary 4.3.

Corollary 4.10 established below is an example of application of Theorem 4.1 with $H$ different from $X$. Prior to its formulation, we introduce a definition.

${ }^{*}$ See Section 2.3 . 
Definition 4.8. Let $K$ be a wedge in $X$ and $f$ be an element from $X$. An operator $A: X \rightarrow X$ is said to be $f$-bounded with respect to $K$ if, for every $x \in X$, there exists a constant $\beta \in(-\infty,+\infty)$ such that

$$
-\beta f \leqq_{K} A x \leqq_{K} \beta f .
$$

In other words, $A$ is $f$-bounded if the element $A x$ is $f$-measurable for all $x$. It follows from Proposition 2.11 that, in the pathological cases where $0 \leqq_{K} f \leqq_{K} 0$ or $f$ is incomparable with zero, every operator $A$ which is $f$-bounded with respect to $K$ has the property im $A \subseteq K^{\diamond}$.

Remark 4.9. An operator $f$-bounded with respect to $K$ is, in particular, $f$-bounded from above in the sense of the definition from [5], Chapt. 2, 1 . The converse statement is not true.

Corollary 4.10. Let $f \geqq_{K} 0$ be a given element and $A_{k}: X \rightarrow X, k=1,2$, be completely continuous linear operators leavfing the wedge $K$ invariant, satisfying condition (4.1), and $f$-bounded with respect to $K$.

Then the existence of a non-negative constant $\alpha$ for which relation (3.2) is satisfied implies estimate (4.3) for the spectral radius of the operator $A_{1}-A_{2}$.

Proof. Setting

$$
H:=X_{K}(f),
$$

we see that condition (3.4) is satisfied due to the $f$-boundedness of $A_{1}$ and $A_{2}$ with respect to $K$. By Lemma 2.14, the element $f$ satisfies relation (2.8) and, hence,

$$
f \varepsilon_{K ; X_{K}(f)} 0 .
$$

Therefore, condition (3.3) holds with $H$ given by (4.7), and it remains to apply Theorem 4.1 .

\section{IMPORTANCE OF CONDITION (4.1)}

As is seen from the proof of Theorem 4.1, the applicability of statements on $K$ substantial eigenvalues is guaranteed by condition (4.1). It is natural to expect that estimating the spectrum of an operator on the base of assumptions of type (3.2) is not possible any more if one admits the existence of a non-zero eigenvalue which is not $K$-substantial, and imposes no additional conditions on $A$.

The following example [11] shows that the assumption on the fulfilment of condition (4.1)in Theorem 4.1 is essential and, generally speaking, cannot be omitted.

Example 5.1. Let us consider the set

$$
K=\left\{\left(\begin{array}{l}
x_{1} \\
x_{2}
\end{array}\right): x_{1} \geq 0, x_{2} \in \mathbb{R}\right\} .
$$

Obviously, $K$ is a solid wedge in $X:=\mathbb{R}^{2}$, and the blade of $K$ has the form

$$
K^{\diamond}=\left\{\left(\begin{array}{l}
0 \\
c
\end{array}\right): c \in \mathbb{R}\right\} \text {. }
$$


It is not difficult to verify that the linear operator $A$ given by the matrix $A=$ $\left(\begin{array}{ll}a_{11} & a_{12} \\ a_{21} & a_{22}\end{array}\right)$ leaves invariant the set $K$ given by (5.1) if, and only if

$$
a_{11} \geq 0, \quad a_{12}=0 .
$$

Furthermore, one can show that, under condition (5.2), $A$ vanishes on $K^{\diamond}$ if, and only if

$$
a_{22}=0 .
$$

A vector $f=\left(\begin{array}{l}f_{1} \\ f_{2}\end{array}\right)$ belongs to the interior of $K$ if, and only if

$$
f_{1}>0 .
$$

whereas the corresponding condition (4.5) is equivalent to the inequality $\alpha f_{1} \geq a_{11} f_{1}$, which, in view of (5.4), means that

$$
\alpha \geq a_{11} .
$$

If condition (2.65) or, which is the same in our case, equality (5.3) is violated, then assumption (4.5), generally speaking, cannot guarantee the validity of the estimate $r(A) \leq \alpha$ for the spectral radius of $A$. Indeed, it is clear from (5.2) that $r(A)=$ $\max \left\{a_{11},\left|a_{22}\right|\right\}$ and, hence,

$$
r(A) \geq\left|a_{22}\right| .
$$

However, if the inequalities

$$
\left|a_{22}\right|>\alpha \geq a_{11} \geq 0
$$

hold, then the assertion of Corollary 4.5 in the case considered would have the form $r(A) \leq \alpha$, which is impossible in view of (5.6) and (5.7).

Thus, condition (2.65) Corollary 4.5 (and, therefore, condition (4.1) in Theorem 4.1), generally speaking, cannot be dropped.

\section{UNIQUE SOLVABILITY OF LINEAR EQUATIONS}

Theorem 4.1 allows one to obtain efficient conditions under which the linear equation

$$
x=A_{1} x-A_{2} x+q,
$$

where $A_{1}$ and $A_{2}$ are linear operators, possesses a unique solution for an arbitrary element $q$ from $X$.

Corollary 6.1. Let $X$ be a real Banach space, $K \subset X$ be a wedge, and $A_{i}: X \rightarrow X$, $i=1,2$, be completely continuous linear operators leaving $K$ invariant and satisfying condition (4.1). In addition, assume that relation (3.2) is satisfied with some constant $\alpha \in[0,1)$ and element $f \in X$ possessing property (4.2).

Then equation (6.1) is uniquely solvable for arbitrary $q \in X$, and its solution $x$ is represented by the convergent Neumann series

$$
x=\sum_{k=0}^{+\infty}\left(A_{1}-A_{2}\right)^{k} q .
$$


Proof. It suffices to notice that, by virtue of Theorem 4.1, the conditions assumed guarantee that the spectrum of the operator $A_{1}-A_{2}$ is contained in the closure of the unit disk in $\mathbb{C}$.

In the cases where the fact of convergence of series (6.2) is less important, one may prefer to use the following

Corollary 6.2. Let $K$ be a proper wedge in a real Banach space $X$ and $A_{i}: X \rightarrow X$, $i=1,2$, be bounded linear operators leaving $K$ invariant and satisfying condition (4.1). Assume that relation (3.2) is true, where $\alpha \in[0,1)$ and $f \in X$ is an element relation (4.2) is satisfied.

Then the homogeneous equation

$$
x=A_{1} x-A_{2} x
$$

has no non-trivial solutions. If, moreover, the operators $A_{1}$ and $A_{2}$ are such that $A_{1}-A_{2}$ is a Fredholm operator of index 0 , then equation (6.1) is uniquely solvable for an arbitrary $q \in X$.

Proof. In view of assumption (4.1) and Lemma 2.48, it follows from Theorem 3.1 that operator (3.6) has no eigenvalues outside the open interval $(-1,1)$ and, in particular, the number 1 is not an eigenvalue for the operator mentioned. Therefore, zero is the unique solution of the homogeneous equation (6.3). The unique solvability of equation (6.1) for any $q$ is guaranteed by the assumption that $A_{1}-A_{2}$ is a Fredholm operator of index 0 .

Corollary 6.2 allows one to obtain the following statement.

Corollary 6.3. Let $K$ be a proper wedge in a real Banach space $X$ and $A_{i}: X \rightarrow X$, $i=1,2$, be bounded linear operators leaving $K$ invariant, satisfying the condition

$$
K^{\diamond} \subseteq \operatorname{ker} A_{1} \cap \operatorname{ker} A_{2} .
$$

Assume also that relation (3.2) is true, where $\alpha \in[0,1)$ is a constant and $f \in X$ is an element possessing property (4.2). Then the homogeneous equation

$$
x=\sigma_{1} A_{1} x+\sigma_{2} A_{2} x
$$

has no non-trivial solutions for any values $\left\{\sigma_{1}, \sigma_{2}\right\} \subset\{-1,1\}$.

If, in addition, for some $\left\{\sigma_{1}, \sigma_{2}\right\} \subset\{-1,1\}$, the mapping $\sigma_{1} A_{1}+\sigma_{2} A_{2}$ is a Fredholm operator of index 0 , then the equation

$$
x=\sigma_{1} A_{1} x+\sigma_{2} A_{2} x+q,
$$

is uniquely solvable for an arbitrary $q \in X$.

Proof. Let us define the operators $\tilde{A}_{i}: X \rightarrow X, i=1,2$, by putting

$$
\tilde{A}_{1}:=\frac{1+\sigma_{1}}{2} A_{1}+\frac{1+\sigma_{2}}{2} A_{2}
$$


and

$$
\tilde{A}_{2}:=\frac{1-\sigma_{1}}{2} A_{1}+\frac{1-\sigma_{2}}{2} A_{2}
$$

One can verify that the relations

$$
\sigma_{1} A_{1}+\sigma_{2} A_{2}=\tilde{A}_{1}-\tilde{A}_{2}
$$

and

$$
A_{1}+A_{2}=\tilde{A}_{1}+\tilde{A}_{2}
$$

are true. It is also easy to see from (6.7) and (6.8) that both operators $\tilde{A}_{1}$ and $\tilde{A}_{2}$ leave invariant the wedge $K$.

Assumption (6.4) guarantees the fulfilment of the inclusion

$$
K^{\diamond} \subseteq \operatorname{ker}\left(\tilde{A}_{1}-\tilde{A}_{2}\right) \text {. }
$$

Moreover, by virtue of (6.9), condition (3.2) can be rewritten as

$$
\tilde{A}_{1} f+\tilde{A}_{2} f \leqq_{K} \alpha f .
$$

We have thus shown that Corollary 6.2 can be applied with $A_{1}$ and $A_{2}$ replaced by $\tilde{A}_{1}$ and $\tilde{A}_{2}$, respectively.

\section{AN EXAMPLE OF A LINEAR INTEGRAL EQUATION}

We illustrate the idea of the results above on an example. Let us consider the problem on the continuous solutions of the equation

$$
x(t)=\int_{0}^{1} h(t, s) x(\omega(s)) d s+q(t), \quad t \in[0,1],
$$

where $q:[0,1] \rightarrow \mathbb{R}$ is continuous, the function $\omega:[0,1] \rightarrow[0,1]$ is measurable, $h(t, \cdot):[0,1] \rightarrow \mathbb{R}$ is Lebesgue integrable for all $t \in[0,1]$, and $h(\cdot, s):[0,1] \rightarrow \mathbb{R}$ is continuous for almost every $s$ from $[0,1]$.

7.1. The general argument deviation. The techniques of Section 4 allow one toobtain the following theorem.

Theorem 7.1. Let there exist a non-negative continuous function $\psi:[0,1] \rightarrow \mathbb{R}$ such that

$$
\underset{t \in[0,1] \backslash \Gamma_{\psi, \omega}}{\operatorname{vrai} \max _{t}} \frac{1}{\psi(\omega(t))} \int_{0}^{1}|h(\omega(t), s)| d s<+\infty
$$

and

$$
h(\omega(t), s)=0 \quad \text { for a. e. } t \in \Gamma_{\psi, \omega} \text { and a. e. } s \in[0,1] \text {, }
$$

where

$$
\Gamma_{\psi, \omega}:=\{t \in[0,1] \mid \psi(\omega(t))=0\} .
$$


Moreover, assume that the inequality

$$
\underset{t \in[0,1] \backslash \Gamma_{\psi, \omega}}{\operatorname{vrai} \max } \frac{1}{\psi(\omega(t))} \int_{0}^{1}|h(\omega(t), s)| \psi(\omega(s)) d s<1
$$

is satisfied.

Then equation (7.1) possesses a unique solution for any continuous function $q$ : $[0,1] \rightarrow \mathbb{R}$.

Proof. Equation (7.1) can obviously be rewritten in form (6.1), where the operators $A_{i}, i=1,2$, in the Banach space $X:=C([0,1], \mathbb{R})$ of all the continuous scalar functions on $[0,1]$ are introduced by the formulae

$$
\left(A_{i} x\right)(t):=\int_{0}^{1} \max \left\{(-1)^{i+1} h(t, s), 0\right\} x(\omega(s)) d s, \quad t \in[0,1], i=1,2,
$$

for any continuous $x:[0,1] \rightarrow \mathbb{R}$. Clearly, each of these operators leaves invariant the wedge

$$
\mathscr{K}_{\omega}:=\{u \in C([0,1], \mathbb{R}) \mid u(\omega(t)) \geq 0 \text { for a. e. } t \in[0,1]\} .
$$

It is easy to show that mappings (7.6) are completely continuous linear operators possessing property (4.1) with respect to the wedge $K=\mathscr{K}_{\omega}$.

Let $\mathscr{C}_{\psi, \omega}$ be the set of all the continuous functions $x:[0,1] \rightarrow \mathbb{R}$ for which there exists a non-negative constant $\beta_{x}$ such that the estimate

$$
|x(\omega(t))| \leq \beta_{x} \psi(\omega(t))
$$

is true for a. e. $t \in[0,1]$.

It is easy to see that $\mathscr{C}_{\psi, \omega}$ is a linear manifold in $C([0,1], \mathbb{R})$. Assumptions (7.2) and (7.3) and formula (7.6) imply that, for any $x$ from $C([0,1], \mathbb{R})$, the estimate

$$
\left|\left(A_{1} x\right)(\omega(t))-\left(A_{2} x\right)(\omega(t))\right| \leq \psi(\omega(t)) \Delta \max _{\xi \in[0,1]}|x(\xi)|
$$

is true at a. e. $t$ from $[0,1] \backslash \Gamma_{\psi, \omega}$, where

$$
\Delta:=\underset{t \in[0,1] \backslash \Gamma_{\psi, \omega}}{\operatorname{vrai} \max } \frac{1}{\psi(\omega(t))} \int_{0}^{1}|h(\omega(t), s)| d s .
$$

Indeed, let $x \in C([0,1], \mathbb{R})$ be arbitrary. According to (7.6), we obtain

$$
\begin{aligned}
& \left|\left(A_{1} x\right)(\omega(t))-\left(A_{2} x\right)(\omega(t))\right|=\left|\int_{0}^{1} h(\omega(t), s) x(\omega(s)) d s\right| \\
& \quad \leq \max _{\xi \in[0,1]}|x(\xi)| \int_{0}^{1}|h(\omega(t), s) x(\omega(s))| d s
\end{aligned}
$$

for a. e. $t \in[0,1]$. For almost all $t \notin \Gamma_{\psi, \omega}$, we have $\psi(\omega(t)) \neq 0$ and, thus, by (7.8),

$$
\int_{0}^{1}|h(\omega(t), s) x(\omega(s))| d s \leq \Delta \psi(\omega(t))
$$


which, together with (7.9), yields (7.7). Moreover, assumption (7.3) guarantees that

$$
\left(A_{1} x\right)(\omega(t))=\left(A_{2} x\right)(\omega(t))
$$

for a. e. $t \in \Gamma_{\psi, \omega}$. Therefore, estimate (7.7) is true almost for all $t$ from the entire interval $[0,1]$, not only those lying outside the set $\Gamma_{\psi, \omega}$.

This means that condition (3.4) is satisfied with the above definitions of $A_{1}$ and $A_{2}$ and $H:=\mathscr{C}_{\psi, \omega}$.

Furthermore, the function $\psi$ satisfies the condition

$$
\psi \varepsilon \mathscr{K}_{\omega} ; \mathscr{C}_{\psi, \omega} 0
$$

which means that (3.3) holds with $f:=\psi$ and $K:=\mathscr{K}_{\omega}$. Indeed, according to Definition 2.16, relation (7.11) means that, for any $x$ from $\mathscr{C}_{\psi, \omega}$, there exists a constant $\beta \geq 0$ such that

$$
|x(\omega(t))| \leq \beta \psi(\omega(t))
$$

at almost every point $t$ from the interval $[0,1]$. However, the above property is an immediate consequence of the definition of the set $\mathscr{C}_{\psi, \omega}$.

Finally, condition (7.3) and inequality (7.5) guarantee that

$$
\int_{0}^{1}|h(\omega(t), s)| \psi(\omega(s)) d s \leq \alpha \psi(\omega(t)) \quad \text { for a. e. } t \in[0,1],
$$

where

$$
\alpha:=\underset{t \in[0,1] \backslash \Gamma_{\psi, \omega}}{\operatorname{vrai} \max } \frac{1}{\psi(\omega(t))} \int_{0}^{1}|h(\omega(t), s)| \psi(\omega(s)) d s
$$

and, by (7.5), $\alpha<1$. In view of (7.6), relation (7.12) can be rewritten in form (3.2) for $K:=\mathscr{K}_{\omega}$. Applying Theorem 4.1, we conclude that 1 is a regular value for the operator (3.6) corresponding to the given problem.

The above theorem implies, for example, the following statement.

Corollary 7.2. Assume that, for certain $\tau \in[0,1]$ and $\gamma \geq 0$, the functions $h$ : $[0,1]^{2} \rightarrow \mathbb{R}$ and $\omega:[0,1] \rightarrow[0,1]$ satisfy the conditions

$$
\underset{t \in[0,1] \backslash \omega^{-1}(\tau)}{\operatorname{vrai} \max } \frac{1}{|\omega(t)-\tau|^{\gamma}} \int_{0}^{1}|\omega(s)-\tau|^{\gamma}|h(\omega(t), s)| d s<1,
$$

and

$$
\underset{t \in[0,1] \backslash \omega^{-1}(\tau)}{\operatorname{vrai} \max _{t}} \frac{1}{|\omega(t)-\tau|^{\gamma}} \int_{0}^{1}|h(\omega(t), s)| d s<+\infty .
$$

In the case where

$$
\operatorname{mes} \omega^{-1}(\tau)>0
$$

assume, in addition, that

$$
h(\tau, s)=0 \quad \text { for a.e. } s \in[0,1] .
$$


Then equation (7.1) has a unique solution for any continuous function $q:[0,1] \rightarrow \mathbb{R}$.

Proof. It suffices to apply Theorem 7.1 with

$$
\psi(t):=|t-\tau|^{\gamma}, \quad t \in[0,1]
$$

in which case set (7.4) is given by the formula

$$
\Gamma_{\psi, \omega}=\{t \in[0,1] \mid \omega(t)=\tau\} .
$$

Assumption (7.3) of the theorem mentioned is satisfied in this case. Indeed, if the set $\omega^{-1}(\tau)$ has zero measure, then property (7.3) is obvious, whereas in the case where (7.15) is true, condition (7.3) is satisfied due to assumption (7.16).

Remark 7.3. If the function $\omega:[0,1] \rightarrow[0,1]$ possesses the property

$$
\underset{t \in[0,1]}{\operatorname{vrai} \min }|\omega(t)-\tau|>0,
$$

then condition (7.14) of Corollary 7.2 is a consequence of its assumption (7.13). Indeed, it follows from (7.13) and (7.17) that

$$
\begin{aligned}
& \underset{\operatorname{vrai} \max }{\operatorname{man} 1] \backslash \omega^{-1}(\tau)} \frac{1}{|\omega(t)-\tau|^{\gamma}} \int_{0}^{1}|h(\omega(t), s)| d s \\
& \quad \leq \frac{1}{\varepsilon^{\gamma}} \underset{t \in[0,1] \backslash \omega^{-1}(\tau)}{\operatorname{vraimax}} \frac{1}{|\omega(t)-\tau|^{\gamma}} \int_{0}^{1}|\omega(s)-\tau|^{\gamma}|h(\omega(t), s)| d s<\frac{1}{\varepsilon^{\gamma}},
\end{aligned}
$$

where $\varepsilon:=$ vrai $\min _{t \in[0,1]}|\omega(t)-\tau|$. Therefore, relation (7.14) is true.

Remark 7.4. Condition (7.13) of Corollary 7.2 is unimprovable in the sense that the corresponding non-strict inequality

$$
\underset{t \in[0,1] \backslash \omega^{-1}(\tau)}{\operatorname{vrai} \max } \frac{1}{|\omega(t)-\tau|^{\gamma}} \int_{0}^{1}|\omega(s)-\tau|^{\gamma}|h(\omega(t), s)| d s \leq 1 .
$$

does not guarantee the unique solvability of equation (7.20) for all continuous $q$. In order to show this, it is sufficient to consider the simplest functional equation

$$
x(t)=x(\vartheta)+q(t), \quad t \in[0,1],
$$

where $\vartheta$ is a given point from $[0,1]$ and $q:[0,1] \rightarrow \mathbb{R}$ is a continuous function. Obviously, equation (7.19) can be rewritten as (7.1) with $\omega(s):=\vartheta$ and $h(t, s):=1$ for all $t$ and almost every $s$ from [0,1]. Equation (7.19) has no continuous solutions for any continuous function $q:[0,1] \rightarrow \mathbb{R}$ satisfying the inequality $q(\vartheta) \neq 0$. Nevertheless, the corresponding condition (7.18) is true in the form of an equality with arbitrary $\vartheta \neq \tau$ and non-negative $\gamma$. Note that, for $\gamma=1$, one can also refer to the example of equation (7.28) from Remark 7.7. 
7.2. The case of a power transformation of argument. For instance, in the case of the equation

$$
x(t)=\int_{0}^{1} h(t, s) x\left(s^{\alpha}\right) d s+q(t), \quad t \in[0,1]
$$

where $\alpha \in(0,+\infty)$ is a constant and $q:[0,1] \rightarrow \mathbb{R}$ is a continuous function, the following statement is true.

Corollary 7.5. Assume that there exists some $\tau \in[0,1]$ for which the conditions

$$
\sup _{t \in[0,1] \backslash\{\tau\}} \frac{1}{|t-\tau|^{\gamma}} \int_{0}^{1}\left|s^{\alpha}-\tau\right|^{\gamma}|h(t, s)| d s<1
$$

and

$$
\sup _{t \in[0,1] \backslash\{\tau\}} \frac{1}{|t-\tau|^{\gamma}} \int_{0}^{1}|h(t, s)| d s<+\infty
$$

are satisfied with a certain $\gamma \in[0,+\infty)$. Then equation (7.20) is uniquely solvable for any continuous function $q:[0,1] \rightarrow \mathbb{R}$.

Proof. Obviously, assumption (7.21) implies that

$$
\sup _{t \in[0,1] \backslash\{\sqrt[\alpha]{\tau\}}]} \frac{1}{\left|t^{\alpha}-\tau\right|^{\gamma}} \int_{0}^{1}\left|s^{\alpha}-\tau\right|^{\gamma}\left|h\left(t^{\alpha}, s\right)\right| d s<1,
$$

which means that condition (7.13) is satisfied with

$$
\omega(t):=t^{\alpha}, \quad t \in[0,1] .
$$

In view of (7.22), condition (7.14) holds with $\omega$ given by (7.23). Thus, Corollary 7.2 can be applied.

The following statement gives somewhat simpler but more restrictive conditions sufficient for the solvability of equation (7.20).

Corollary 7.6. Equation (7.20) has a unique continuous solution for any continuous $q$, provided that the inequality

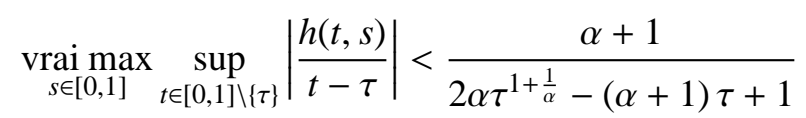

is satisfied with some $\tau \in[0,1]$.

Proof. In view of (7.24), there exists a $\delta \in[0,1)$ such that

$$
\left|\frac{h(t, s)}{t-\tau}\right| \leq \frac{\delta(\alpha+1)}{2 \alpha \tau^{1+\frac{1}{\alpha}}-(\alpha+1) \tau+1}
$$


for almost every $s \in[0,1]$ and all $t \in[0,1] \backslash\{\tau\}$. Using estimate (7.25) and taking into account the identity

$$
\int_{0}^{1}\left|\xi^{\alpha}-\tau\right| d \xi=\frac{2 \alpha \tau^{1+\frac{1}{\alpha}}+1}{\alpha+1}-\tau
$$

we conclude that

$$
\frac{1}{|t-\tau|} \int_{0}^{1}\left|\xi^{\alpha}-\tau\right||h(t, \xi)| d \xi \leq \int_{0}^{1}\left|\xi^{\alpha}-\tau\right| d \xi \text { vrai max } \max \left|\frac{h(t, s)}{t-\tau}\right| \leq \delta<1
$$

for every $t$ different from $\tau$, i. e., condition (7.21) is satisfied with $\gamma:=1$. Moreover, by virtue of (7.25), condition (7.22) is satisfied with this value of $\gamma$. Applying Corollary 7.5, we obtain the required assertion.

Corollary 7.6 implies, in particular, that equation (7.20) is uniquely solvable if

$$
\text { vrai } \max \sup _{t \in[0,1]} t^{-1}|h(t, s)|<\alpha+1 .
$$

It should be noted that (7.27) is weaker than the condition

$$
\text { vrai } \max \sup _{t \in[0,1]} t^{-1}|h(t, s)|<1,
$$

which is obtained by using the standard techniques (e. g., Theorem 5.5 of [7] with $E=C([0,1], \mathbb{R}), K$ defined as the cone of non-negative functions, $y_{0} \equiv 1$, and the operator $A$ given by the expression in the right-hand side of (7.20)).

Remark 7.7. None of conditions (7.24) and (7.27) can be weakened. Indeed, consider the equation

$$
x(t)=\frac{(\alpha+1)|t-\tau|}{2 \alpha \tau^{1+\frac{1}{\alpha}}-\tau(\alpha+1)+1} \int_{0}^{1} x\left(s^{\alpha}\right) d s, \quad t \in[0,1],
$$

where $\alpha \in(0,+\infty)$ and $\tau \in[0,1]$ are arbitrary constants. Obviously, equation (7.28) has form (7.20) with

$$
h(t, s):=\frac{(\alpha+1)|t-\tau|}{2 \alpha \tau^{1+\frac{1}{\alpha}}-\tau(\alpha+1)+1}
$$

for all $t$ and almost every $s$ from $[0,1]$. Moreover, due to formulae (7.26) and (7.29), we have

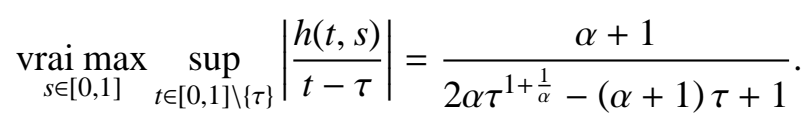

However, the homogeneous equation (7.28) has the non-trivial solution

$$
x(t)=\frac{(\alpha+1)|t-\tau|}{2 \alpha \tau^{1+\frac{1}{\alpha}}-\tau(\alpha+1)+1}, \quad t \in[0,1] .
$$

Thus, we see that condition (7.24) is unimprovable. In order to show the optimality of condition (7.27), it is sufficient to put $\tau:=0$ in (7.28). 


\section{REFERENCES}

[1] Birкhoff, G.: Lattice Theory, Amer. Math. Soc., Providence, R. I., 1948.

[2] Dunford, N. and Schwartz, J. T.: Linear Operators. Part I, John Wiley \& Sons Inc., New York, 1988.

[3] Jentzsch, P.: Über Integralgleichungen mit positivem Kern, J. Reine Angew. Math., 141 (1912), 235-244.

[4] Kantorovich, L. V. and Akilov, G. P.: Functional Analysis, 2nd edn., Pergamon Press, Oxford, 1982.

[5] Krasnoselskit, M. A.: Positive Solutions of Operator Equations, Noordhoff, Groningen, 1964.

[6] Krasnoselskit, M. A., Lifshits, E. A., and Sobolev, A. V.: Positive Linear Systems. Method of Positive Operators, Heldermann, Berlin, 1989.

[7] Krasnoselskit, M. A., Vainikko, G. M., Zabreiko, P. P., Rutitskit, Y. B., and Stetsenko, V. Y.: Approximate Solution of Operator Equations, Noordhoff, Groningen, 1972.

[8] Krein, M.: Propriétés fondamentales des ensembles coniques normaux dans l'espace de Banach, C. R. (Doklady) Acad. Sci. URSS (N.S.), 28 (1940), 13-17.

[9] Krein, M. and KreIn, S.: On an inner characteristic of the set of all continuous functions defined on a bicompact Hausdorff space, C. R. (Doklady) Acad. Sci. URSS (N.S.), 27 (1940), 427-431.

[10] Krein, M. G. and Rutman, M. A.: Linear operators leaving invariant a cone in a Banach space, No. 26 in Amer. Math. Soc. Transl., Amer. Math. Soc., R. I., 1950.

[11] RonTó, A.: On substantial eigenvalues of linear operators leaving invariant a closed wedge, in: Folia FSN Universitatis Masarykianae, Mathematica, vol. 13, Masaryk University, 2003, pp. 235246.

[12] RonTó, A.: Upper bounds for the eigenvalues of compact linear operators in a preordered Banach space, Preprint No. 159, Academy of Sciences of the Czech Republic, Mathematical Institute, Praha, 2005.

[13] Stetsenko, V. Y.: On bounds of the spectra of certain classes of linear operators, Sov. Math. Dokl., 5 (1964), 1090-1093.

[14] VuLikн, B. Z.: Introduction to the theory of partially ordered spaces, Wolters-Noordhoff Scientific Publications Ltd., Groningen, 1967.

[15] Zabreiko, P. P., Krasnoselskit, M. A., and Stetsenko, V. Y.: Bounds for the spectral radius of positive operators, Math. Notes, 1 (1967), 306-310.

\section{Author's address}

\section{Andrei Rontó:}

Mathematical Institute, Academy of Sciences of the Czech Republic, ŽižKova 22, CZ-61662 Brno, Czech Republic

E-mail address: ronto@ipm.cz 